\title{
An Efficient Robust Servo Design for Non-Minimum Phase Discrete-Time Systems with Unknown Matched/Mismatched Input Disturbances
}

\author{
Jason Sheng-Hong Tsai ${ }^{1, ~ *, ~ F a e z e h ~ E b r a h i m z a d e h ~}{ }^{1}$, Yun-You Lin ${ }^{1}$, Shu-Mei Guo ${ }^{2}$, Leang-San Shieh ${ }^{3}$, \\ Yau-Tarng Juang ${ }^{4}$
}

${ }^{1}$ Department of Electrical Engineering, National Cheng Kung University, Tainan, Taiwan, Republic of China

${ }^{2}$ Department of Computer Science and Information Engineering, National Cheng Kung University, Tainan, Taiwan, Republic of China

${ }^{3}$ Department of Electrical and Computer Engineering, University of Houston, Houston, Texas, United States of America

${ }^{4}$ Department of Electrical Engineering, National Central University, Taoyuan, Taiwan, Republic of China

Email address:

shtsai@mail.ncku.edu.tw (Jason Sheng-Hong Tsai)

${ }^{*}$ Corresponding author

\section{To cite this article:}

Jason Sheng-Hong Tsai, Faezeh Ebrahimzadeh, Yun-You Lin, Shu-Mei Guo, Leang-San Shieh, Yau-Tarng Juang. An Efficient Robust Servo Design for Non-Minimum Phase Discrete-Time Systems with Unknown Matched/Mismatched Input Disturbances. Automation, Control and Intelligent Systems. Vol. 5, No. 2, 2017, pp. 14-28. doi: 10.11648/j.acis.20170502.11

Received: February 9, 2017; Accepted: March 1, 2017; Published: March 22, 2017

\begin{abstract}
This paper presents an efficient proportional-plus-integral (PI) current-output observer-based linear quadratic discrete tracker (LQDT) design methodology for the non-minimum-phase (NMP) discrete-time system with equal input and output number, for which the minimalized dynamic system contains the unmeasurable system state and unknown external matched/mismatched input disturbances. Illustrative examples are given to demonstrate the effectiveness of the proposed approach.
\end{abstract}

Keywords: Optimal Linear Quadratic Tracker, State Estimator, Disturbance Estimator, Non-Minimum Phase System, Control Zeros

\section{Introduction}

The unknown input observer (UIO) design methodology involves the state estimation for a dynamic system subject to unknown input excitation [1], in which it may contain internal uncertainties and exogenous loads that cannot be measured or inconvenient to measure. Several developed approaches to simultaneously estimate the system state and unknown input can be referred to [1-4] and in the literature therein. Nevertheless, most UIO design methodologies presented in the early literatures require that the transfer function from the unknown input to the system output is minimum-phase and of relative degree one. Recently, some design methodologies, which exempted the assumptions that the transfer function must be the minimum-phase (with respect to the relation between the unknown input to the system output, but not the relation between the control input to the system output) and unit-relative-degree constraints have been reported in $[1,3,4]$ under some rank conditions. More precisely, in order for the design methodology to be feasible in $[1,3]$, the dimensions of the unknown inputs/disturbances must be no greater than the output dimensions. In addition, the distribution matrix of unknown inputs/disturbances with some pre-specified rank conditions has be known a priori.

It is noticed that, in the real world, either the distribution matrix of unknown inputs/disturbances or the number of unknown inputs might be known, or both might be unknown. Even both are known, they might not satisfy the rank conditions. This implies that there can be more unknown inputs than both the control inputs and measured outputs. Also, noticed that when both the distribution matrix of unknown inputs/disturbances and the number of unknown inputs/ disturbances are unknown, it is difficult to precisely estimate the components of unknown inputs/disturbances. For a given 
controllable and observable continuous-time dynamic system with unknown mismatched inputs/disturbances, the theoretical design methodology has been investigated by She et al. [5], and it has been reported that, for all times, the output of the plant with mismatched disturbances would be identical to the output of the plant with matched disturbances, which were produced via an equivalent input disturbance (EID) entering into the plant through the control input channels. The above fact is called as the (continuous-time) EID principle. Thus, one can use a matched disturbance model as an equivalent of the mismatched disturbance model. As a result, the control objective for this case is to estimate and feedback the effects produced by the EID through the input channels to cancel the negative effects induced by the mismatched disturbance. More detailed comments on improving disturbance-rejection performance based on an EID principle can be found in $[5,6]$. Hence, based on the EID principle, a given NMP continuous-time plant with the mismatched non-minimum-phase disturbance model can be replaced by a given NMP continuous-time plant with the matched non-minimum-phase disturbance model. Nevertheless, neither the unknown input estimation nor the servo design methodology for the NMP plant has been fully addressed in [5, $6]$.

Some new optimal proportional-integral-derivative (PID) filter-shaped PI feedback linear quadratic analog tracker/linear quadratic digital tracker (LQAT/LQDT) design methodologies for non-square NMP continuous-time and discrete-time transfer function matrices and their minimalized dynamic systems are proposed in the recent works [7] and [8], respectively. It is known that for the square NMP plant (with equal input and output number), it is difficult to find a non-singular square transformation matrix to ensure that the transformed closed-loop system becomes a minimum-phase (MP) one (in the sensor of 'control zero'). As a result, the PID-filter shaped PI feedback LQAT/LQDT design methodologies for the square NMP system are left for future research as shown in $[7,8]$. At this point, it must call the attention that the recently proposed approaches [7, 8] still work well for the square and/or non-square MP plants with explicitly known disturbances, but not for the plants with unknown disturbances. An advanced design approach of observer-based optimal tracking controllers for time delay systems with external disturbances can be referred to the recent work in [9]. Also, new simultaneous state and output disturbance estimations for a class of switched linear systems with unknown inputs have been presented by Yang et al. [10]. Some precocious disturbance estimations and disturbance cancellation controller design methodologies can be referred to $[11,12]$. Nevertheless, the design methodologies for the NMP plant have not been addressed in [9-12].

In the real world, an unknown external disturbance usually occurs at the plant input, which would result in poor tracking performance. To overcome this issue, Chang [13] constructed the discrete-time proportional-integral observer (PIO) to develop a state and disturbance estimator for the discrete-time system with an unknown external disturbance to improve its tracking performance. Consequently, an advanced algorithm on the robust discrete-time output tracking controller design for NMP systems has been proposed by Chang et al. [14]. However, some restrictive conditions have been imposed on the existing design methodology in [14], namely: (i) The NMP plant has to be in square dimension; (ii) Only the matched input disturbance is considered; (iii) The variation of the disturbance in two consecutive sampling instants is not changed significantly; (iv) The model-following-based command generator which uses the constant input has to be constructed to generate the desired output; and (v) The use of the past output measurements to simultaneously estimate the system state and its equivalent input disturbance.

In this paper, for the NMP square strictly proper discrete-time transfer function matrix, for which the minimalized dynamic system with unmeasurable system state and its unknown external matched/mismatched input disturbances, we propose a PI current output-based observer (PICO) design methodology to simultaneously estimate the system state and its equivalent input disturbance. In the proposed approach, a variable input signal instead of the constant in [14] is used to generate a drastic command input. Then, a current-output observer-based LQDT with a high-gain property is developed to have the desired tracking performance.

This paper is organized as follows. An efficient PI current-output observer-based LQDT for the NMP discrete-time system with equal input and output number, for which the minimalized dynamic system with unmeasurable system state and unknown external matched/mismatched input disturbances is presented in Sec. 2. Numerical simulations are given in Sec. 3 to demonstrate the effectiveness of the proposed approach. Finally, conclusion is given in Sec. 4.

\section{Current-Output Observer-Based LQDT for NMP Discrete-Time System with an Unknown Disturbance}

Comparison with the results developed in [14], some extensions over [14] are summarized as follows: (i) The use of the past output measurements to estimate the system state with unknown matched input disturbances developed in [14] has been extended to the use of the current output measurements to estimate the system state and the EID principle for the unknown matched/mismatched input disturbances, so that the tracking performance can be rapidly improved; (ii) The restriction on the constant command input of reference model presented in [14] has been relaxed to time-varying command input of reference model, so that a more robust and flexible tracker can be achieved; (iii) The closed-loop poles of the observer error dynamic system presented in this paper are optimally assigned inside a circle with a pre-specified radius $\alpha \quad(0<\alpha \leq 1)$, but not just lying inside the unit circle ( $\alpha=1$ ) presented in [14], so that the transient response of the designed servo can be significantly improved.

To present the discrete-time EID principle, let the dynamic 
discrete-time NMP systems with the mismatched and matched input disturbances $f(k) \in \mathfrak{R}^{n}$ and $d(k) \in \mathfrak{R}^{m}$ be respectively described by

$$
\begin{gathered}
x_{o}(k+1)=G x_{o}(k)+H u(k)+f(k), \\
y(k)=C x_{o}(k)
\end{gathered}
$$

and

$$
\begin{gathered}
x(k+1)=G x(k)+H(u(k)+d(k)), \\
y(k)=C x(k),
\end{gathered}
$$

where $x_{o}(k) \in \mathfrak{R}^{n}, \quad x(k) \in \mathfrak{R}^{n}$ are the state vectors, $u(k) \in \mathfrak{R}^{m}$ is the control input vector, $y(k) \in \mathfrak{R}^{p}$ is the measured output vector, and $d(t) \in \mathfrak{R}^{m}$ are stateindependent disturbance signals. Equations (1)-(2) present the dynamic system with mismatched disturbance at the input, while (3)-(4) present the same dynamic system with matched disturbance. The discrete-time EID principle states that outputs of the plant with mismatched disturbances $f(k)$ in (1) and matched disturbances $H d(k)$ in (3) are identical in a dead-beat sense; however, the states of both models $x_{o}(k)$ and $x(k)$ are not identical if $m<n$.

The discrete-time EID principle can be proved directly as follows. Taking the z-transformations of (1)-(4), one has

$$
\begin{aligned}
\mathrm{y}(z) & =C\left(z I_{n}-G\right)^{-1} H \mathrm{v}(z)+C\left(z I_{n}-G\right)^{-1} f(z) \\
& =C\left(z I_{n}-G\right)^{-1} H \mathrm{v}(z)+C\left(z I_{n}-G\right)^{-1} H d(z),
\end{aligned}
$$

provided that $C\left(z I_{n}-G\right)^{-1} H d(z)=C\left(z I_{n}-G\right)^{-1} f(z) \in \mathfrak{R}^{p}$ holds, where $y(z), u(z)$, and $f(z)$ are z-transformations of $y(k), u(k)$, and $f(k)$, respectively. Since $C\left(z I_{n}-G\right)^{-1} H$

is of dimension $p \times m$, where $p \leq m$, the desired EID $d(z)$ exists theoretically. However, $\left(z I_{n}-G\right)^{-1} H d(z)=\left(z I_{n}-G\right)^{-1} f(z) \in \mathfrak{R}^{n}$ does not hold if $m<n$, since it has more equation numbers than variable $(d(\bullet))$ numbers. More precisely, a linear combination of pre-specified columns of $H$, i.e.

$$
H d(k)=\left[h_{1} h_{2} \ldots h_{m}\right] \times\left[d_{1}(k) d_{2}(k) \ldots d_{m}(k)\right]^{T} \quad=d_{1}(k) h_{1}+
$$
$d_{2}(k) h_{2}+\ldots+d_{m}(k) h_{m} \in \mathfrak{R}^{n} \quad$ (combining with only $m$ pre-specified independent column vectors of dimension $n$ ) does not span the arbitrarily specified vector space $f(k) \in \Re^{n}$ which has $n$ independent signal components, where $h_{i} \in \mathfrak{R}^{n}$ and $d_{i}(k) \in \Re$ for $i=1,2, \ldots, m$. This complete the proof of the discrete-time EID principle.

The objective of this paper is to design (i) a current-output-based state and disturbance observer and (ii) the observer-based optimal tracker, which would ensure that the controlled system in (1)-(4) has a desired tracking performance for a given arbitrary reference trajectory with some drastic variations. In this regards, the following assumptions are pre-assumed throughout the paper.

Assumption $1[13,14]$ : The system (1)-(4) is observable and controllable.

Assumption $2[13,14]$ : The sampling time $T_{S}$ is selected sufficiently small such that the disturbance satisfies $\Delta d(k)=d(k+1)-d(k) \in O\left(T_{S}\right) \forall k$.

Assumption 3 [13, 14]: The condition $\operatorname{rank}\left(\left[\begin{array}{cc}G_{n \times n}-I_{n} & H_{n \times m} \\ C_{p \times n} & 0_{p \times m}\end{array}\right]\right)=n+p \quad$ is satisfied and $\operatorname{det}(\mathrm{CH}) \neq 0$. This condition implies that the dynamic system (1)-(4) has no transmission zeros lying at 'one', which often arises in the response to the robust tracking problem.

The design procedure of the PICO-based LQDT for the square NMP systems with equal input and output number and unknown matched/mismatched input disturbances is described in the following.

Lemma 1 [14]: Suppose that $d(k)$ is bounded and smooth and Assumption 3 holds, then $d(k)$ satisfies the following equations:

$$
\begin{gathered}
d(k+1)-d(k) \in O\left(T_{s}\right), \\
d(k+2)-2 d(k+1)+d(k) \in O\left(T_{s}^{2}\right) .
\end{gathered}
$$

Proof of Lemma 1: See [14, 15].

Then, we offer the following design steps, and each step is developed based on the insights of classical control theory.

Step 1: Specify the structure of the current output-based state estimator and disturbance observer.

Construct a current output-based observer, which uses previous and the current measurements of the output $(y(k)$, $y(k-1), y(k-2), \ldots)$ to predict state and disturbance, without requiring the information on $f(k)$, as follows

$$
\begin{gathered}
\hat{x}(k)=G \hat{x}(k-1)+H u(k-1)+L_{o}[y(k)-\hat{y}(k)] \\
+H\left[L_{2} p_{1}(k-1)+L_{1} p_{2}(k-1)\right], \\
\hat{y}(k)=C \hat{x}(k), \\
p_{1}(k)=p_{1}(k-1)+[y(k)-\hat{y}(k)], \\
p_{2}(k)=p_{2}(k-1)+p_{1}(k-1), \\
\hat{d}(k)=L_{2} p_{1}(k)+L_{1} p_{2}(k),
\end{gathered}
$$

where $\hat{x}(k), \hat{y}(k)$ and, $\hat{d}(k)$ are the estimations of the state vector $x(k)$, output vector $y(k)$, and disturbance $d(k)$, respectively. The parameter matrices $L_{o} \in \mathfrak{R}^{n \times p}$ and $L_{i} \in \Re^{m \times p}$ for $i=1,2$ are to be designed. Physical interpretation of (11) associated with (10) is given by 


$$
\begin{aligned}
d(k+1)-d(k) \cong & {[d(k)-d(k-1)]+[y(k)-\hat{y}(k)] } \\
& -[y(k-1)-\hat{y}(k-1)] \\
\cong & {[d(k)-d(k-1)]+[y(k-1)-\hat{y}(k-1)], }
\end{aligned}
$$

where $p_{2}(k)=d(k+1)-d(k)$ and $p_{1}(k)=y(k)-\hat{y}(k)$.

Step 2: Construct error dynamic equations for state estimator and disturbance observer designs.

i) Derive the state estimation error dynamic equations.

According to Lemma 1, one can obtain

$$
\tilde{x}(k)=\left(G-L_{o} C G\right) \tilde{x}(k-1)+\left(H-L_{o} C H\right) \tilde{d}(k)+O\left(T_{s}^{2}\right),
$$

and derive $\tilde{y}(k)$ as

$$
\begin{aligned}
\tilde{y}(k)= & y(k)-\hat{y}(k) \\
\cong & C\{[G x(k-1)+H(u(k-1)+d(k-1))] \\
& -[G \hat{x}(k-1)+H(u(k-1)+\hat{d}(k-1))]\} \\
= & C[G \tilde{x}(k-1)+H \tilde{d}(k-1)],
\end{aligned}
$$

where $\quad \tilde{d}(k)=d(k)-\hat{d}(k), \quad \tilde{y}(k)=y(k)-\hat{y}(k) \quad$ and $\tilde{x}(k)=x(k)-\hat{x}(k)$ are disturbance estimation error, output estimation error, and state estimation error, respectively.

ii) Derive the disturbance estimation error dynamic equations.

Define two disturbance estimation error variables as

$$
\begin{gathered}
w_{1}(k-1)=d(k)-\left[d(k-1)+L_{1} p_{1}(k-1)\right], \\
\begin{aligned}
w_{2}(k-1)=d(k-1)-\hat{d}(k-1) \\
=d(k-1)-\left[L_{2} p_{1}(k-1)+L_{1} p_{2}(k-1)\right],
\end{aligned}
\end{gathered}
$$

where $w_{1}(k), w_{2}(k) \in \Re^{m}$. From (14)-(16) and (8)-(12), the dynamic equations of $w_{1}(k)$ and $w_{2}(k)$ can be derived as

$$
\begin{aligned}
w_{1}(k)= & d(k+1)-d(k)-L_{1} p_{1}(k) \\
= & d(k+1)-d(k)-L_{1}\left[\tilde{y}(k)+p_{1}(k-1)\right] \\
= & d(k+1)-2 d(k)+d(k-1)-L_{1} C G \tilde{x}(k-1) \\
& \quad-L_{1} C H w_{2}(k-1)+w_{1}(k-1), \\
& \\
w_{2}(k)= & d(k)-L_{1} p_{2}(k)-L_{2} p_{1}(k) \\
= & d(k)-L_{1}\left[p_{1}(k-1)+p_{2}(k-1)\right]-L_{2}\left[p_{1}(k-1)+\tilde{y}(k)\right] \\
= & w_{1}(k-1)+w_{2}(k-1)-L_{2} \tilde{y}(k) \\
= & w_{1}(k-1)+\left(I_{m}-L_{2} C H\right) w_{2}(k-1)-L_{2} C G \tilde{x}(k-1) .
\end{aligned}
$$

iii) Construct the integrated state and disturbance estimation error dynamic equations.

From (13)-(14) and (17)-(18), one has

$$
w(k)=\left[\begin{array}{ccc}
G-L_{o} C G & 0_{n \times m} & H-L_{o} C H \\
-L_{1} C G & I_{m} & -L_{1} C H \\
-L_{2} C G & I_{m} & I_{m}-L_{2} C H
\end{array}\right] w(k-1)+\left[\begin{array}{c}
O\left(T_{s}^{2}\right) \\
d(k+1)-2 d(k)+d(k-1) \\
0_{m \times 1}
\end{array}\right]
$$

$$
\begin{gathered}
=(\bar{G}-L \bar{C}) w(k-1)+O\left(T_{s}^{2}\right), \\
\tilde{y}(k)=\bar{C} w(k),
\end{gathered}
$$

where

$$
w(k)=\left[\begin{array}{lll}
\tilde{x}^{T}(k) & w_{1}^{T}(k) & w_{2}^{T}(k)
\end{array}\right]^{T} \in \Re^{n+2 m},
$$

$L=\left[\begin{array}{lll}L_{o}^{T} & L_{1}^{T} & L_{2}^{T}\end{array}\right]^{T}, \quad \bar{C}=\left[\begin{array}{lll}C G & 0_{p \times m} C H\end{array}\right] \quad, \quad$ and $\bar{G}=\left[\begin{array}{ccc}G & 0_{n \times m} & H \\ 0_{m \times n} & I_{m} & 0_{m \times m} \\ 0_{m \times n} & I_{m} & I_{m}\end{array}\right]$.

Step 3: Perform the optimal linear quadratic observer design.

To have a desired observer gain $L$ in terms of $\left(L_{o}, L_{1}, L_{2}\right)$ for the current output-based observer in (8)-(12) such that the closed-loop observer error dynamic system poles are optimally assigned inside a circle with a pre-specified radius $\alpha \quad(0<\alpha \leq 1)$, let us perform the following transformations $\tilde{G}=\bar{G} / \alpha, \quad \tilde{C}=\bar{C} / \alpha$, and $0<\alpha \leq 1$, which yield to a transformed equation as

$$
\begin{aligned}
w_{t}(k) & =\frac{1}{\alpha}(\bar{G}-L \bar{C}) w_{t}(k-1)+O\left(T_{s}^{2}\right) \\
& =(\tilde{G}-L \tilde{C}) w_{t}(k-1)+O\left(T_{s}^{2}\right) .
\end{aligned}
$$

Then, solving the following steady-state algebraic Riccati equation,

$$
P=\tilde{G} P \tilde{G}^{T}-\left(\tilde{G} P \tilde{C}^{T}\right)\left(R_{o}+\tilde{C} P \tilde{C}^{T}\right)^{-1}\left(\tilde{G} P \tilde{C}^{T}\right)^{T}+Q_{o},
$$

yields the desired observer gain as

$$
L=\left(\tilde{G} P \tilde{C}^{T}\right)\left(R_{o}+\tilde{C} P \tilde{C}^{T}\right)^{-1},
$$

where the weighting matrices $Q_{o} \in \mathfrak{R}^{(n+2 m) \times(n+2 m)}$ and $R_{o} \in \Re^{p \times p}$ are selected with a high-gain property. Hence, the eigenvalues of $(\tilde{G}-L \tilde{C})$ are guaranteed to be inside the unit circle. The obtained observer gain $L$ in (23) is then applied to the original observer system in (8)-(12), which results in the closed-loop characteristic equation

$$
\begin{aligned}
& \operatorname{det}\left[z I_{(n+2 m)}-(\bar{G}-L \bar{C})\right] \\
= & \operatorname{det}\left[z I_{(n+2 m)}-(\tilde{G}-L \tilde{C}) \times \alpha\right]=0 .
\end{aligned}
$$

The equality in (24) implies that the eigenvalues of $(\bar{G}-L \bar{C})$ are equal to those of $(\tilde{G}-L \tilde{C})$ multiplied by the factor $\alpha$. Clearly, it is desirable to choose a small value of $\alpha$, which would speed up the convergence of the estimation errors [16].

Step 4: Specify the structure of the proportional-plus-integral (PI) model-following servo control design.

i) Realize the command generator tracker.

To proceed the PICO-based tracker design, let the reference 
output $y_{m}(k) \in \mathfrak{R}^{p} \quad$ be generated by the following square/non-square minimum phase reference model

$$
\begin{aligned}
x_{m}(k+1) & =\left(G_{m}+I_{\kappa}\right) x_{m}(k)+H_{m} u_{m}(k) \\
& =\bar{G}_{m} x_{m}(k)+H_{m} u_{m}(k), \\
y_{m}(k) & =C_{m} x_{m}(k), x_{m}(0)=C_{m}^{\dagger} y(0),
\end{aligned}
$$

so that $y_{m}(k) \rightarrow r_{m}(k)$ and expect the controlled system output $y(k) \rightarrow y_{m}(k) \rightarrow r_{m}(k)$ as well for any command input $r_{m}(k)$, in which

$$
\begin{gathered}
u_{m}(k)=-K_{m} x_{m}(k)+E_{m} r_{m}(k+1), \\
K_{m}=\left(R_{m}+H_{m}^{T} P_{m} H_{m}\right) H_{m}^{T} P_{m} \bar{G}_{m}, \\
E_{m}=\left(R_{m}+H_{m}^{T} P_{m} H_{m}\right)^{-1}\left[H_{m}^{T}\left(I_{\kappa}-\left(\bar{G}_{m}-H_{m} K_{m}\right)^{T}\right) C_{m}^{T}\right]^{-1} Q_{m},
\end{gathered}
$$

where $n \geq \kappa \geq m \geq l \geq p$. In addition, $\quad x_{m}(k) \in \mathfrak{R}^{\kappa}$ and $u_{m}(k) \in \mathfrak{R}^{l}$ are the state and input vectors of the reference model, respectively. Furthermore, the weighting matrices $Q_{m} \in \Re^{p \times p}$ and $R_{m} \in \Re^{l \times l}$ are selected with a high-gain property, and $P_{m} \in \Re^{\kappa \times \kappa}$ is obtained via solving the following Riccati equation for servo control design $[8,16]$

$$
\begin{gathered}
\bar{G}_{m}^{T} P_{m} \bar{G}_{m}-P_{m}-\bar{G}_{m}^{T} P_{m} H_{m}\left(H_{m}^{T} P_{m} H_{m}+R_{m}\right)^{-1} H_{m}^{T} P_{m} \bar{G}_{m} \\
+C_{m}^{T} Q_{z} C_{m}=0 .
\end{gathered}
$$

ii) Perform the proportional model-following servo control design.

If Assumption 3 holds, system (1)-(4) has no transmission zeros lying at 'one', then one can obtain

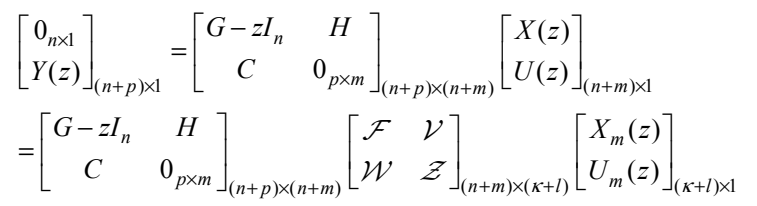

(representing $X(z)$ and $U(z)$ as the weighted feedbacks of $X_{m}(z)$ and $\left.X_{m}(z)\right)$

$$
\begin{gathered}
=\left[\begin{array}{cc}
\mathcal{F}_{n \times \kappa} & \boldsymbol{0}_{n \times p} \\
\boldsymbol{0}_{p \times \kappa} & I_{p \times p}
\end{array}\right]_{(n+p) \times(\kappa+p)}\left[\begin{array}{c}
0_{\kappa \times 1} \\
Y_{m}(z)
\end{array}\right]_{(\kappa+p) \times 1} \\
=\left[\begin{array}{cc}
\mathcal{F}_{n \times \kappa} & \boldsymbol{0}_{n \times p} \\
\boldsymbol{0}_{p \times \kappa} & I_{p \times p}
\end{array}\right]_{(n+p) \times(\kappa+p)}\left[\begin{array}{cc}
\left(G_{m}+I_{\kappa}\right)-z I_{\kappa} & H_{m} \\
C_{m} & 0_{p \times l}
\end{array}\right]_{(\kappa+p) \times(\kappa+l)}\left[\begin{array}{c}
X_{m}(z) \\
U_{m}(z)
\end{array}\right]_{(\kappa+l) \times 1}
\end{gathered}
$$

(matching the dimension of the Rosenbrock system matrix of the pre-assigned MP system in (25)-(26) with the one of the controlled NMP system in (1)-(4), without alternating transmission zeros of the pre-assigned MP system in (25)-(26) subject to $n \geq \kappa)$

$$
=\left[\begin{array}{cc}
\mathcal{F} G_{m} & \mathcal{F} H_{m} \\
C_{m} & 0_{p \times l}
\end{array}\right]\left[\begin{array}{l}
X_{m}(z) \\
U_{m}(z)
\end{array}\right] ; \quad z=1,
$$

(an intermediary MP model to be matched at the steady state)

where $\mathcal{F} \in \mathfrak{R}^{n \times \kappa}, \quad \mathcal{V} \in \mathfrak{R}^{n \times l}, \mathcal{W} \in \mathfrak{R}^{m \times \kappa}$, and $\mathcal{Z} \in \mathfrak{R}^{m \times l}$ are undetermined parameter matrices [14]. Since Assumption 3 holds, from (31)-(32) it can be observed that

$\left[\begin{array}{cc}\mathcal{F} & \mathcal{V} \\ \mathcal{W} & \mathcal{Z}\end{array}\right]=\left[\begin{array}{cc}G-I_{n} & H \\ C & 0\end{array}\right]^{-1}\left[\begin{array}{cc}\mathcal{F} G_{m} & \mathcal{F} H_{m} \\ C_{m} & 0\end{array}\right]=\left[\begin{array}{cc}\Omega_{11} & \Omega_{12} \\ \Omega_{21} & \Omega_{22}\end{array}\right]\left[\begin{array}{cc}\mathcal{F} G_{m} & \mathcal{F} H_{m} \\ C_{m} & 0\end{array}\right]$,

where $\quad \Omega_{11} \in \mathfrak{R}^{n \times n}, \Omega_{12} \in \mathfrak{R}^{n \times p}, \Omega_{21} \in \mathfrak{R}^{p \times n}, \quad$ and $\Omega_{22} \in \mathfrak{R}^{p \times p}$. Then, by given the known matrices $G_{m}, C_{m}$, $\Omega_{11}$, and $\Omega_{12}$, we can obtain the solution for the matrix $\mathcal{F}$ as the solution of Lyapunov equation $\mathcal{F} G_{m}^{-1}-\Omega_{11} \mathcal{F}=\Omega_{12} C_{m} G_{m}^{-1} \quad$ [17]. Hence, matrices $\mathcal{F}, \mathcal{V}, \mathcal{W}$, and $\mathcal{Z}$ can be obtained.

iii) Derive error dynamic equations for the PI model-following servo control design.

Based on the pre-designed parameters $\mathcal{F}, \mathcal{V}, \mathcal{W}$, and $\mathcal{Z}$ presented in Step ii), one can then derive error dynamic equations for the PI model-following servo control design as follows. Let

$$
\begin{gathered}
e_{x}(k)=x(k)-\left[\mathcal{F} x_{m}(k)+\mathcal{V} u_{m}(k)\right], \\
e_{y}(k)=y(k)-y_{m}(k) .
\end{gathered}
$$

Then, substituting (1)-(4) and (25)-(26) into (35) yields

$$
\begin{aligned}
e_{x}(k+1)= & x(k+1)-\left[\mathcal{F} x_{m}(k+1)+\mathcal{V} u_{m}(k+1)\right] \\
= & G x(k)+H u(k)-\mathcal{F}\left[\left(G_{m}+I_{\kappa}\right) x_{m}(k)+H u_{m}(k)\right] \\
& -\mathcal{V} u_{m}(k+1)+H d(k)+O\left(T_{s}^{2}\right) \\
= & G\left[x(k)-\mathcal{F} x_{m}(k)-\mathcal{V} u_{m}(k)\right]+G \mathcal{F} x_{m}(k)+G \mathcal{V} u_{m}(k) \\
& +H\left[u(k)-\mathcal{W} x_{m}(k)-\mathcal{Z} u_{m}(k)\right] \\
& -H\left[\mathcal{W} x_{m}(k)+\mathcal{Z} u_{m}(k)\right]-\mathcal{F} x_{m}(k) \\
& -\mathcal{F} G_{m} x_{m}(k)-\mathcal{F} H_{m} u_{m}(k)-\mathcal{V}\left[u_{m}(k+1)-u_{m}(k)\right] \\
& -\mathcal{V} u_{m}(k)+H d(k)+O\left(T_{s}^{2}\right) \\
= & G e_{x}(k)+H\left[u(k)-\mathcal{W} x_{m}(k)-\mathcal{Z} u_{m}(k)\right] \\
+ & {\left[\left(G-I_{n}\right) \mathcal{F}+H \mathcal{W}-\mathcal{F} G_{m}\right] x_{m}(k) } \\
+ & {\left[\left(G-I_{n}\right) \mathcal{V}+H \mathcal{Z}-\mathcal{F} H_{m}\right] u_{m}(k) } \\
& -\mathcal{V}\left[u_{m}(k+1)-u_{m}(k)\right]+H d(k)+O\left(T_{s}^{2}\right) .
\end{aligned}
$$

Since the first row components of (34) show $\left(G-I_{n}\right) \mathcal{F}+H \mathcal{W}=\mathcal{F} G_{m}$ and $\left(G-I_{n}\right) \mathcal{V}+H \mathcal{Z}=\mathcal{F} H_{m}$, one has 


$$
\begin{aligned}
e_{x}(k+1)= & G e_{x}(k)+H\left[u(k)-\mathcal{W} x_{m}(k)-\mathcal{Z} u_{m}(k)+d(k)\right] \\
& -\mathcal{V}\left[u_{m}(k+1)-u_{m}(k)\right]+O\left(T_{s}^{2}\right) .
\end{aligned}
$$

Also,

$$
\begin{gathered}
e_{y}(k)=C x(k)-C_{m} x_{m}(k) \\
=C\left[x(k)-\left(\mathcal{F} x_{m}(k)+\mathcal{V} u_{m}(k)\right)\right] \\
=C e_{x}(k) .
\end{gathered}
$$

Now, define the integral of $\left[y(k)-y_{m}(k)\right]$ as another state variable

$$
\eta(z)=\frac{1}{z-1}\left[Y(z)-Y_{m}(z)\right]
$$

By taking the inverse z-transform, from (40) one has

$$
\eta(k+1)=\eta(k)+\left[y(k)-y_{m}(k)\right]=\eta(k)+C e_{x}(k) .
$$

Error dynamic equations for the PI model-following servo control design can then be formulated as

$$
\begin{gathered}
z(k+1)=G_{z} z(k)+H_{z}\left[u(k)-\mathcal{W} x_{m}(k)-\mathcal{Z} u_{m}(k)+d(k)\right] \\
-\mathcal{V}\left[u_{m}(k+1)-u_{m}(k)\right]+O\left(T_{s}^{2}\right), \\
e_{y}(k)=C_{z} z(k),
\end{gathered}
$$

and $C_{z}=\left[\begin{array}{ll}C & 0\end{array}\right]$.

Step 5: Perform the PICO-based optimal LQDT design.

By neglecting the last two terms in the right hand side of (42), replacing the unknown $d(k)$ by the estimated $\hat{d}(k)$, replacing the unmeasurable $x(k)$ by the estimated $\hat{x}(k)$, and performing the transformations $\tilde{G}_{z}=G_{z} / \beta, \tilde{H}_{z}=H_{z} / \beta$, in which $0<\beta \leq 1$, the optimal control law is given by

$$
u(k)=-K_{1} \hat{e}_{x}(k)-K_{2} \eta(k)-\hat{d}(k)+\mathcal{W} x_{m}(k)+\mathcal{Z} u_{m}(k),
$$

where

$$
\begin{gathered}
\hat{e}_{x}(k)=\hat{x}(k)-\mathcal{F} x_{m}(k)-\mathcal{V} u_{m}(k), \\
K=\left[\begin{array}{c:c}
K_{1 m \times n} & K_{2 m \times p}
\end{array}\right]=\left(\tilde{H}_{z}^{T} P_{z} \tilde{H}_{z}+R_{z}\right)^{-1} \tilde{H}_{z}^{T} P_{z} \tilde{G}_{z},
\end{gathered}
$$

in which $P_{z}$ is the positive definite solution of the following algebraic Riccati equation

$$
\tilde{G}_{z}^{T} P_{z} \tilde{G}_{z}-P_{z}-\tilde{G}_{z}^{T} P_{z} \tilde{H}_{z}\left(\tilde{H}_{z}^{T} P_{z} \tilde{H}_{z}+R_{z}\right)^{-1} \tilde{H}_{z}^{T} P_{z} \tilde{G}_{z}+Q_{z}=0 .
$$

Finally, the structure of the PICO-based LQDT for the square system with an unknown external disturbance is shown in Figure 1.

where $\quad z(k)=\left[\begin{array}{c}e_{x}(k) \\ \eta(k)\end{array}\right] \in \Re^{n+p}, \quad G_{z}=\left[\begin{array}{cc}G & 0 \\ C & I_{p}\end{array}\right], H_{z}=\left[\begin{array}{c}H \\ 0\end{array}\right]$,

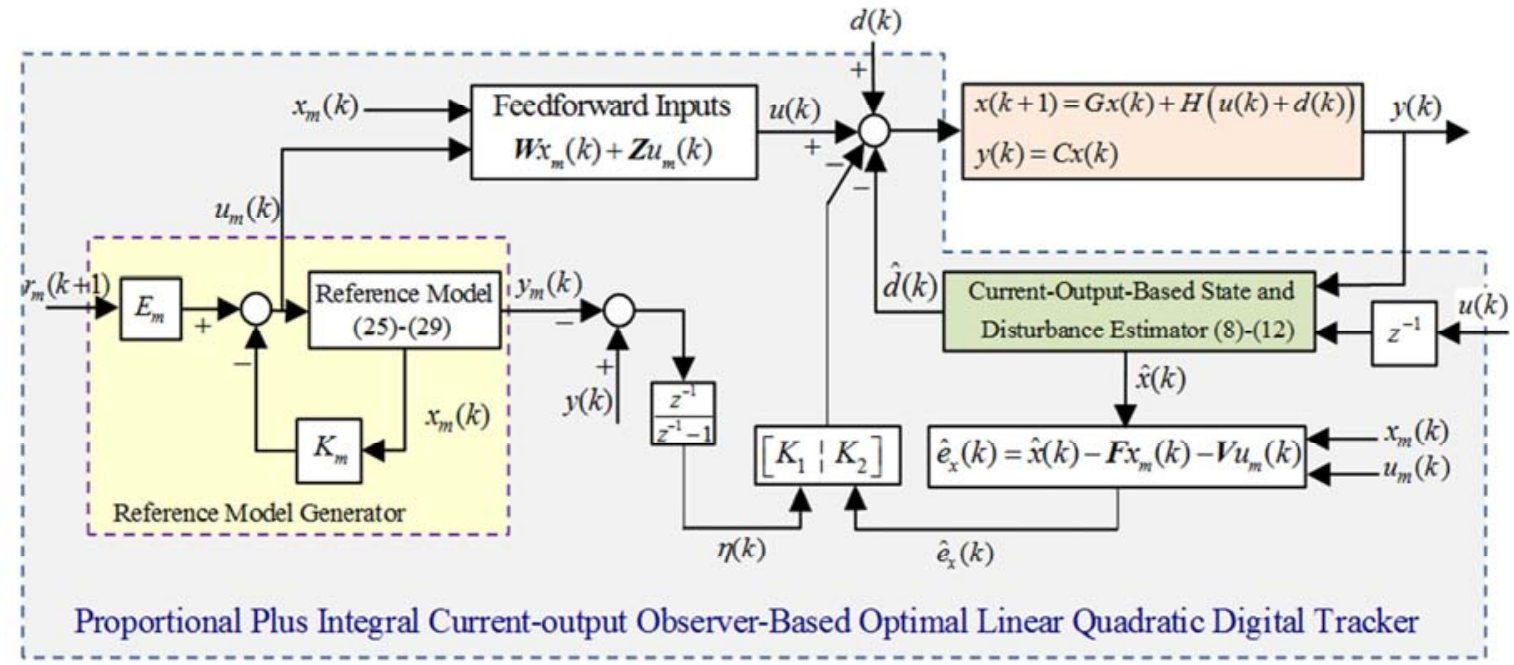

Figure 1. PICO-based optimal LQDT for the discrete-time system with an unknown external disturbance.

Remark 1: If the pair $(\bar{G}, \bar{C})$ is observable, the matrix $(\bar{G}-L \bar{C})$ can be stabilized. That is, an observer gain $L$ can be found such that all eigenvalues of the matrix $(\bar{G}-L \bar{C})$ are lying inside the unit circle. As for the observability condition for the pair $(\bar{G}, \bar{C})$ is concerned, we may state it in Lemma 2 .
Lemma 2: Under Assumptions 1 to 3, the pair $(\bar{G}, \bar{C})$ is observable and the estimation errors are constrained in the small region of $O\left(T_{S}^{2}\right)$.

Proof of Lemma 2: By Popov-Belevitch-Hautus rank test [13], the pair $(\bar{G}, \bar{C})$ is observable if and only if the matrix 
$\left[\begin{array}{c}\lambda I_{n+2 m}-\bar{G} \\ \bar{C}\end{array}\right]$ has full column rank (i.e.,
$\operatorname{rank}\left(\left[\begin{array}{c}\lambda I_{n+2 m}-\bar{G} \\ \bar{C}\end{array}\right]\right)=n+2 m$ ) for all $\lambda \in \mathrm{C}$. Hence, it can be observed that

$$
\begin{aligned}
& \operatorname{rank}\left(\left[\begin{array}{c}
\lambda I_{n+2 m}-\bar{G} \\
\bar{C}
\end{array}\right]\right)=\operatorname{rank}\left(\left[\begin{array}{ccc}
\lambda I_{n}-G & 0_{n \times m} & -H \\
0_{m \times n} & (\lambda-1) I_{m} & 0_{m \times m} \\
0_{m \times n} & -I_{m} & (\lambda-1) I_{m} \\
\hdashline C G & 0_{p \times m} & C H
\end{array}\right]\right) \\
& =\left\{\begin{array}{l}
\operatorname{rank}\left(\left[\begin{array}{c}
\lambda I_{n}-G \\
C G
\end{array}\right]\right)+2 m, \quad \lambda \neq 1 \\
\operatorname{rank}\left(\left[\begin{array}{cc}
G-I_{n} & H \\
C G & C H
\end{array}\right]\right)+m, \quad \lambda=1
\end{array}\right.
\end{aligned}
$$

From (48), one can conclude that the pair $(\bar{G}, \bar{C})$ is observable if Assumption 3 holds. In addition, the estimation errors will be constrained in the small region of $O\left(T_{S}^{2}\right)$, since the sampling time $T_{S}$ is assumed to be sufficiently small.

\section{Illustrative Examples}

In this section, three numerical examples are given to illustrate the proposed approach.

Example 1: Consider the practical discrete-time non-minimum-phase MIMO flight control model considered in [16] with unknown external disturbances in (3), where

$$
G=\left[\begin{array}{rrrrrrr}
0.9805 & 0.0005 & 0.0007 & -0.0000 & -0.0494 & -0.0000 & 0.0000 \\
0.0000 & 0.9948 & 0.0000 & -0.1141 & 0.0000 & 0.0000 & 0.0000 \\
0.0000 & 0.0011 & 0.9988 & -0.0001 & 0.0283 & 0.0000 & 0.0000 \\
0.0000 & 0.0050 & 0.0000 & 0.9931 & 0.0000 & 0.0000 & 0.0000 \\
0.0001 & -0.0000 & -0.0050 & 0.0000 & 0.9989 & 0.0002 & 0.0000 \\
0.0050 & 0.0000 & 0.0000 & -0.0000 & -0.0001 & 1.0000 & 0.0000 \\
0.0000 & 0.0050 & 0.0000 & -0.0003 & 0.0000 & 0.0000 & 1.0000
\end{array}\right] \text {, }
$$

$$
H=\left[\begin{array}{rrr}
-0.2269 & -0.0378 & -0.0000 \\
0.0000 & 0.0000 & -0.1413 \\
-0.0046 & -0.0325 & -0.0001 \\
0.0000 & 0.0000 & -0.0012 \\
0.0000 & 0.0001 & 0.0000 \\
-0.0006 & -0.0001 & -0.0000 \\
0.0000 & 0.0000 & -0.0004
\end{array}\right], C=\left[\begin{array}{lllllll}
0 & 0 & 0 & 0 & 1 & 0 & 0 \\
0 & 0 & 0 & 0 & 0 & 1 & 0 \\
0 & 0 & 0 & 0 & 0 & 0 & 1
\end{array}\right],
$$

with the (unstable) open-loop system poles $\{1,1,0.9939 \pm 0.0238 i, 0.9807,0.9988 \pm 0.0120 i\}$ and the (unstable) 'control zeros' $\{-1.1600,-0.9936,-0.9981,0.9941\}$ $[7,8]$. The initial conditions and sampling time are given $x(0)=\left[\begin{array}{lllllll}0.1 & 0 & 0 & 0.1 & 0 & 0 & 0\end{array}\right]^{T}, \hat{x}(0)=\left[\begin{array}{lllllll}0 & 0 & 0 & 0 & 0 & 0 & 0\end{array}\right]^{T}$, and $T_{s}=0.005 \mathrm{~s}$, respectively. The pole-zero distribution of this open-loop system, i.e. seven poles are located at or near 1, one unstable zero, and one zero (0.9941) are located near 1, makes the robust servo control design more challenging (see Assumption 3). Assume the system state is not measurable. The high-frequency unknown input disturbances are generated by

$d(k)=\left[\begin{array}{c}-1.2 \cos (30 \pi k)-\sin (10 \pi k)-0.8 \cos (5 \pi k)-0.6 \sin (10 / 3 \pi k)-0.4 \cos (2.5 \pi k) \\ -1.2 \cos (15 \pi k)-\sin (5 \pi k)-0.8 \cos (2.5 \pi k)-0.6 \sin (5 / 3 \pi k)-0.4 \cos (1.25 \pi k) \\ -0.5 \cos (10 \pi k)-\sin (3 \pi k)-0.7 \cos (15 \pi k)-0.3 \sin (10 / 3 \pi k)-0.2 \cos (5 \pi k)\end{array}\right]$.

The design procedure is given as follows.

Step 1: Perform the optimal linear quadratic observer design.

Choose an appropriate weighting matrix pair $\left\{Q_{o}, R_{o}\right\}=\left\{10^{8} I_{3}, I_{3}\right\}$ and the radius $\alpha=0.07$ to have the observer gain

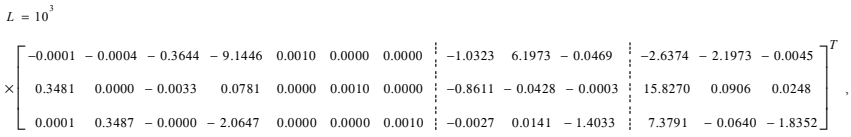

which results in the closed-loop eigenvalues of the observer error-dynamics system at

$$
\sigma(\bar{G}-L \bar{C})=\{0.9940,0.0068 \pm 0.0018 \mathrm{i}, 0.0038 \pm 0.0003 \mathrm{i}
$$

$0.0048 \pm 0.0010 \mathrm{i}, 0.0055 \pm 0.0005 \mathrm{i}, 0.0056,0,0,0\}$. The performance of the proposed discrete-time current output-based estimator is depicted in Figure 2. It shows that the system state $x(k)$ and unknown external disturbance $d(k)$ are well estimated, which implies $\hat{y}(t) \rightarrow y(t)$ and $\hat{d}(t) \rightarrow d(t)$ also.

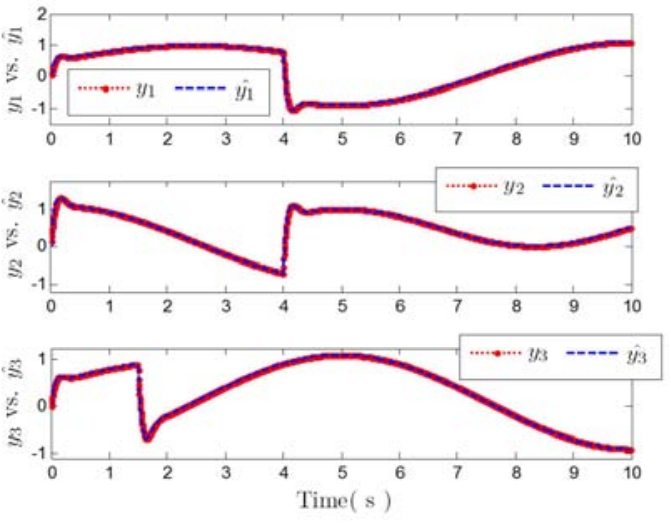

(a)

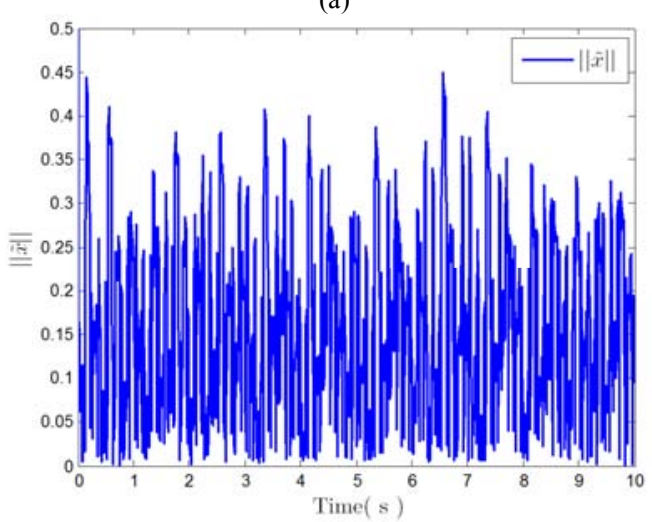

(b) 

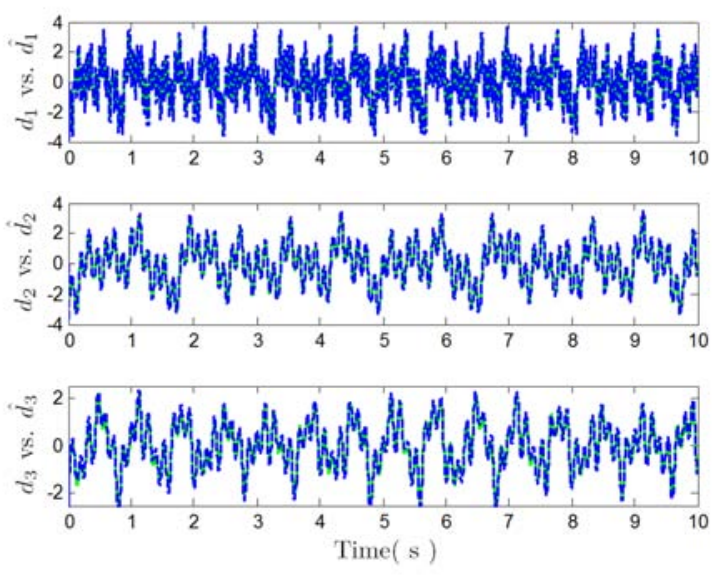

(c)
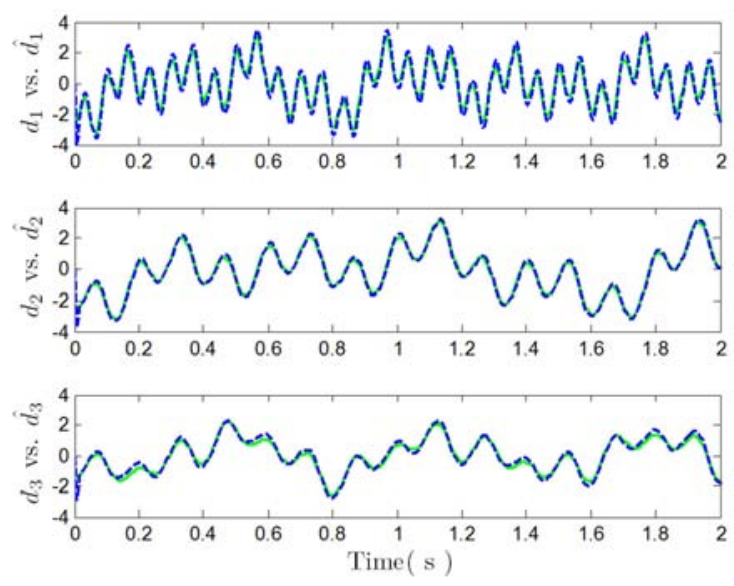

(d)

Figure 2. The closed-loop responses of the PI observer for $i=1 \sim 3:$ (a) $y_{i}(k)$ vs. $\hat{y}_{i}(k)$, (b) estimation errors of the system state; $\|\tilde{x}\|, \quad(c)$ $d_{i}(k)$ vs. $d_{i}(k)$ in global scope; $(d) d_{i}(k)$ vs. $\hat{d}_{i}(k)$ in local scope: up to 2 $s$.

Step 2: Construct the command reference model and solutions of undetermined parameter matrices.

The reference model is given by

$$
\begin{aligned}
& x_{m}(k+1)=\left[\begin{array}{cccc}
0.01 & 0 & 0 & 0 \\
0 & 0.0700 & -0.0320 & 0 \\
0 & 0.0313 & 0 & 0 \\
0 & 0 & 0 & 0.05
\end{array}\right] x_{m}(k)+\left[\begin{array}{ccc}
2 & 0 & 0 \\
0 & 1 & 0 \\
0 & 0 & 0 \\
0 & 0 & 2
\end{array}\right] u_{m}(k), \\
& y_{m}(k)=C_{m}=\left[\begin{array}{cccc}
1.3847 & 0 & 0 & 0 \\
0 & -1.3499 & 0.0432 & 0 \\
0 & 0 & 0 & 1.5175
\end{array}\right] x_{m}(k),
\end{aligned}
$$

with the eigenvalues $\{0.0200,0.0500,0.0100,0.0500\}$ and a finite control zero $10^{-3}$, so that $y_{m}(k) \rightarrow r_{m}(k)$, for the desired command input $r_{m}(k)=\left[r_{m, 1}(k) r_{m, 2}(k) r_{m, 3}(k)\right]^{T}$, where

$$
\begin{aligned}
& r_{m, 1}(k)=\left\{\begin{array}{ll}
0.5 \sin (0.2 \pi k)+0.5, & 0 \leq k<4 \\
\cos (0.2 \pi k)+0.1, & 4 \leq k<10
\end{array},\right. \\
& r_{m, 2}(k)=\left\{\begin{array}{ll}
\cos (0.2 \pi k)+0.1, & 0 \leq k<4 \\
-0.5 \sin (0.3 \pi k)+0.5, & 4 \leq k<10
\end{array},\right. \\
& r_{m, 3}(k)=\left\{\begin{array}{ll}
0.5 \sin (0.2 \pi k)+0.5, & 0 \leq k<1.5 \\
-\cos (0.2 \pi k)+0.1, & 1.5 \leq k<10
\end{array},\right. \\
& \begin{aligned}
u_{m}(k)= & -\left[\begin{array}{rrrr}
0.0050 & -0.0000 & -0.0000 & 0.0000 \\
0.0000 & 0.0690 & -0.0320 & -0.0000 \\
0.0000 & 0.0000 & 0.0000 & 0.0250
\end{array}\right] x_{m}(k) \\
& +\left[\begin{array}{rrr}
0.3611 & 0.0000 & -0.0000 \\
-0.0000 & -0.7408 & 0.0000 \\
-0.0000 & -0.0000 & 0.3295
\end{array}\right] r_{m}(k+1),
\end{aligned}
\end{aligned}
$$

for the weighting matrices $Q_{m}=10^{5} I_{3}$ and $R_{m}=I_{3}$. Then, by given the known matrices $G_{m}, C_{m}, \Omega_{11}$, and $\Omega_{12}$ in (34), we calculate the matrices $\mathcal{F}, \mathcal{V}, \mathcal{W}$, and $\mathcal{Z}$ as

$$
\mathcal{F}=\left[\begin{array}{cccc}
-0.0409 & 470.7045 & 15.1612 & 0.0445 \\
0 & 0 & 0 & -549.6157 \\
506.0737 & 35.5533 & 2.7705 & 0.1016 \\
0 & 0 & 0 & -1.7388 \\
1.3847 & 0 & 0 & 0 \\
0 & -1.349 & 0.0432 & 0 \\
0 & 0 & 0 & 1.5175
\end{array}\right],
$$$$
\mathcal{V}=10^{3} \times\left[\begin{array}{ccc}
0.0001 & -0.5061 & -0.0001 \\
0 & 0 & 1.1571 \\
-1.0229 & -0.0383 & -0.0002 \\
0 & 0 & 0.0037 \\
0 & 0 & 0 \\
0 & 0 & 0 \\
0 & 0 & 0
\end{array}\right] \text {, }
$$

$$
\begin{aligned}
& \mathcal{W}=10^{4} \times\left[\begin{array}{cccc}
-0.2626 & 0.1759 & 0.0115 & 0.0001 \\
1.5755 & 0.0764 & 0.0104 & -0.0007 \\
0 & 0 & 0 & -0.3674
\end{array}\right], \\
& \mathcal{Z}=10^{4} \times\left[\begin{array}{ccc}
0.5304 & -0.1894 & -0.0001 \\
-3.1825 & -0.0824 & 0.0015 \\
0 & 0 & 0.7734
\end{array}\right] .
\end{aligned}
$$

Step 3: Realize the PICO-based optimal LQDT design. Realize the optimal control law

$$
u(k)=-K_{1} \hat{e}_{x}(k)-K_{2} \eta(k)-\hat{d}(k)+\mathcal{W} x_{m}(k)+\mathcal{Z} u_{m}(k),
$$

for the appropriate weighting matrix pair $\left\{Q_{z}, R_{z}\right\}=\left\{10^{5} I_{10}, I_{3}\right\}$ and the radius $\beta=1$, where from (22) the PI state-feedback is

$K=\left[\begin{array}{rrrrrrr:rrr}-4.6632 & 0.0017 & 5.5094 & -0.0000 & -106.8625 & -90.6368 & -0.0371 & -5.0437 & -4.2270 & -0.0017 \\ 0.7654 & -0.0168 & -33.0510 & 0.0000 & 638.2899 & 2.3725 & 0.3658 & 29.9360 & 0.2138 & 0.0169 \\ 0.0000 & -7.3857 & -0.0000 & 0.7899 & 0.0010 & 0.0000 & -145.0735 & 0.0001 & 0.0000 & -6.7310\end{array}\right]$.

The eigenvalues of the closed-loop error-dynamics system

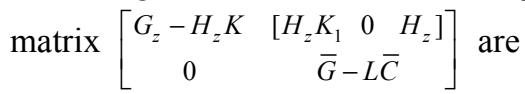


$\left\{0.1849 \times 10^{-4}, 0.4980 \times 10^{-4}, 0.0010,0.9497 \pm 0.0478 i\right.$,

$0.9503 \pm 0.0472 i, 0.9499 \pm 0.0474 i, 0.994,0.9940$,

$0.0068 \pm 0.0018 i, 0.0038 \pm 0.0003 i, 0.0055 \pm 0.0005 i$,

$$
0.0056,0,0,0\} \text {. }
$$

Since $\sigma\left(G_{z}-H_{z} K\right)=\left\{0.1849 \times 10^{-4}, 0.4980 \times 10^{-4}, 0.0010\right.$, $0.9497 \pm 0.0478 i, 0.9503 \pm 0.0472 i, 0.9499 \pm 0.0474 i, 0.994\} \quad$ and $\sigma(\bar{G}-L \bar{C})=\{0.9940,0.0068 \pm 0.0018 i, 0.0038 \pm 0.0003 i$, $0.0055 \pm 0.0005 i, 0.0056,0,0,0\}$, it follows that the separation principle is valid and the stability is guaranteed.

The tracking response and control input of the closed-loop system are shown in Figure 3, although the system of interest is NMP and under disturbances, the system output $y(k)$ well tracks the reference trajectory $y_{m}(k)$.
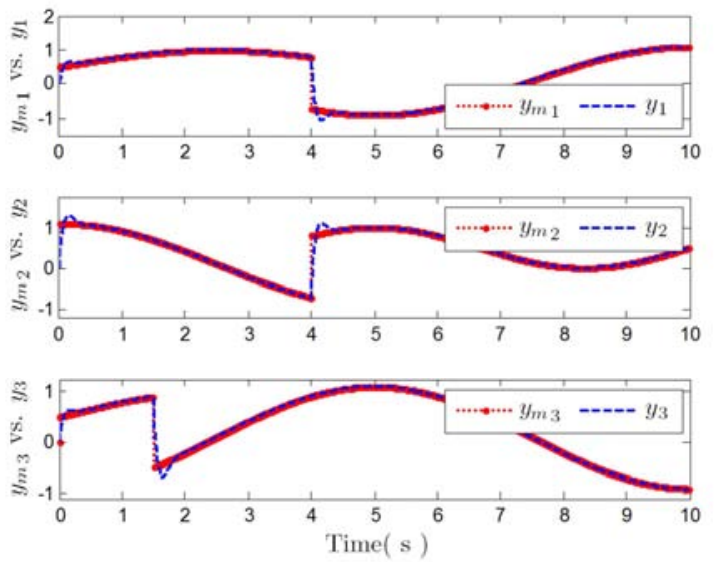

(a)
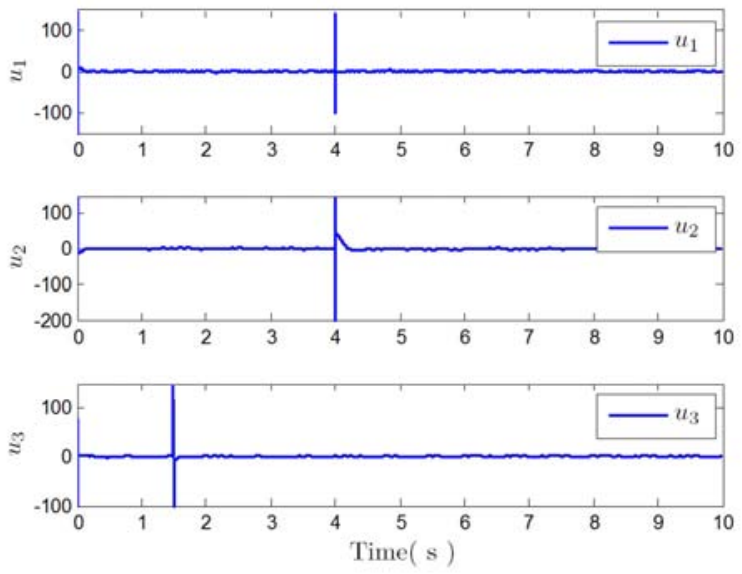

(b)

Figure 3. The closed-loop responses of the system with unknown matched disturbances for $i=1 \sim 3$ : (a) tracking response $y_{i}(k)$ vs. $y_{m} i(k)$, (b) control input $u_{i}(k)$.

In Figure 4, we compare the estimation and tracking performances of two observers obtained by the proposed approach and by Chang et al. method in [14], where the closed-loop eigenvalues of the observer error-dynamics system in [14] are

$$
\sigma\left(\bar{G}-L_{C h} \bar{C}\right)=\{0.9444 \pm 0.0857 i, 0.9542 \pm 0.0755 i
$$

$0.9723 \pm 0.0453 i, 0.8998,0.9475,0.9168,0.9941,10^{-5}, 10^{-5}$, $\left.10^{-5}\right\}$ for the appropriate weighting matrix pair $\left\{Q_{o}, R_{o}\right\}=\left\{10^{5} I_{13}, I_{3}\right\}$. The comparison shows that our proposed method obviously outperforms that in [14].

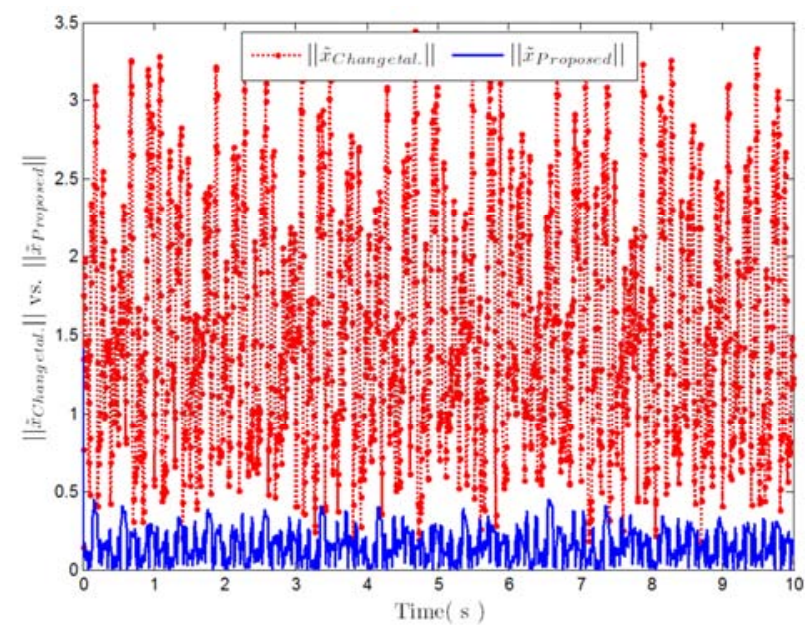

(a)

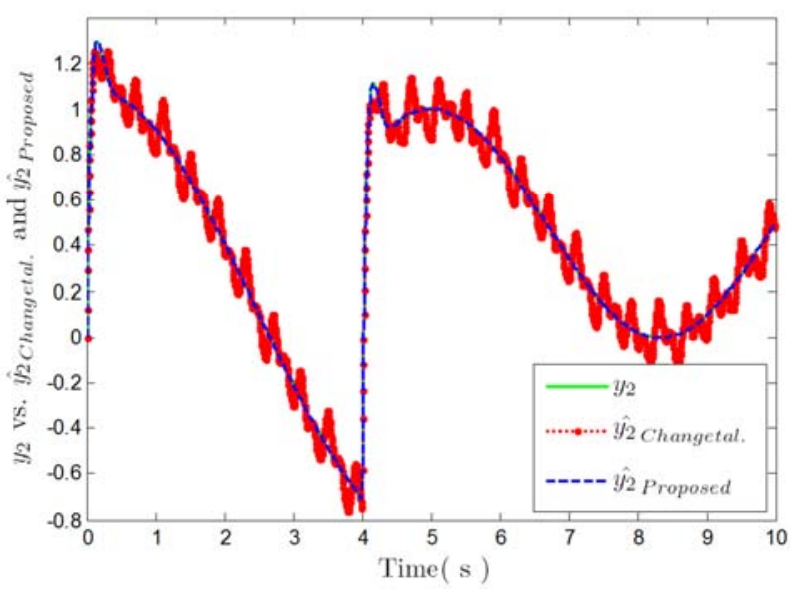

(b)

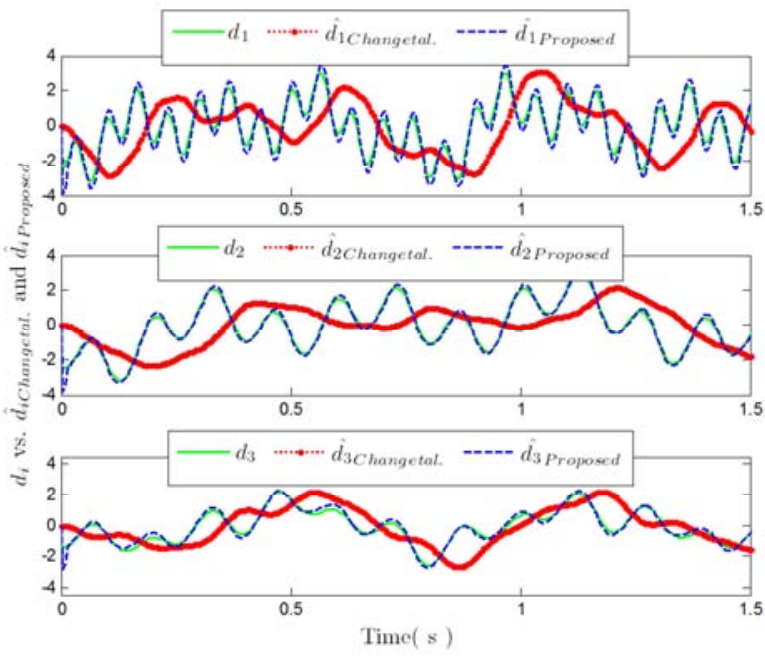

(c) 


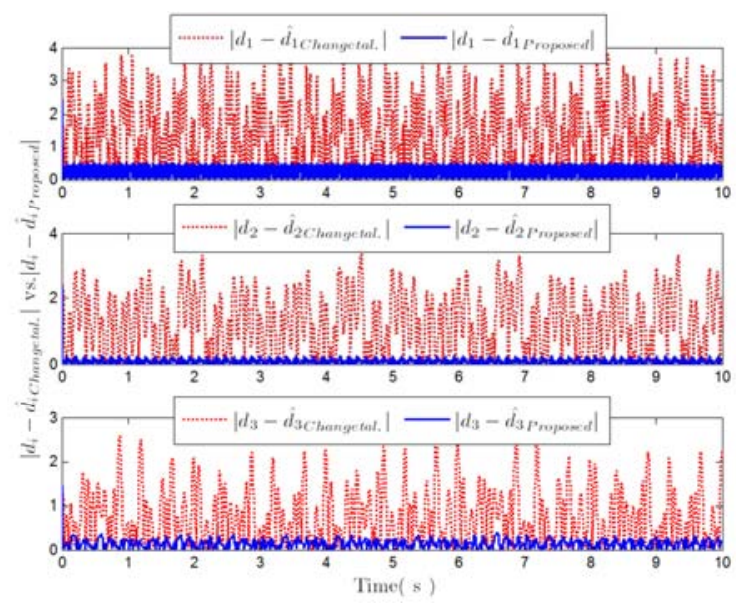

(d)

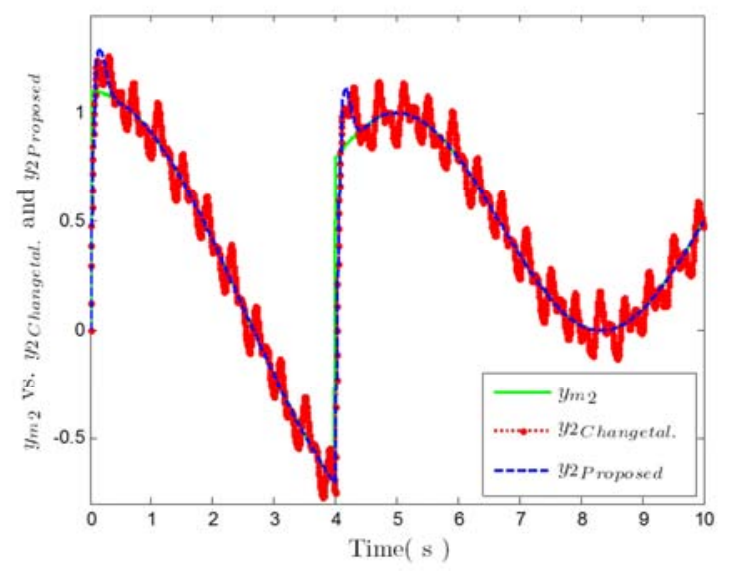

(e)

Figure 4. Comparison between [14] (denoted by the subscript Changetal.) and the proposed method with matched disturbances for $i=1 \sim 3:(a)$ $\left\|\tilde{x}_{\text {Changetal. }}\right\|$ vs. $\left\|\tilde{x}_{\text {Proposed }}\right\|, \quad$ (b) $y_{2}(k)$ vs. $\hat{y}_{2}$ Chngetal. $(k)$ and $\hat{y}_{2 \text { Proposed }}(k)$ (shown by parts: up to $1.5 s$ ), (c) $d_{i}(k)$ vs. $\hat{d}_{i}$ Chngetal. $(k)$ and $\hat{d}_{i \text { Proposed }}(k), \quad(d) \mid d_{i}-\hat{d}_{i}$ Changetal. $\mid$ vs. $\left|d_{i}-\hat{d}_{i \text { Proposed }}\right|, \quad(e)$ tracking responses $y_{m_{2}}(k)$ vs. $\quad y_{2}$ Chngetal. $(k)$ and $y_{2}$ Proposed $(k)$ (shown by parts).

Example 2: Consider the same open-loop system given in Example 1 with the mismatched disturbance $f(k) \in \mathfrak{R}^{n}$, $t=k T_{s}, T_{s}=0.005 \mathrm{~s}$ as follows

$$
f(k)=\left[\begin{array}{c}
0.2 \\
\sin (6 \pi k)-0.4 \cos (2.5 \pi) \\
0.2 \cos (5 \pi k) \\
0.3 \sin (7 \pi k) \\
0.1 \cos (5 \pi k)-0.2 \cos (6.5 \pi k) \\
0.5 \cos (7.5 \pi k) \\
\sin (5 \pi k)
\end{array}\right]
$$

where $f(k) \in \Re^{n}$ has more unknown inputs than the control inputs and measured outputs and the distribution matrix of unknown inputs $(D)$ is unknown. Then, apply the proposed approach to the system of interest. The tracking responses and control inputs of the closed-loop system are shown in Figure 5.

Figure 5 demonstrates that although the system has not only unknown mismatched disturbances but also an unstable zero, parts of the system output $y(k)$ well track the corresponding command inputs of $y_{m}(k)$. However, for the third component, there is still room for improvement. Besides, the comparisons on the estimation performances and tracking responses of two observers depicted in Figure 6 show that our proposed method obviously outperforms that in [14].
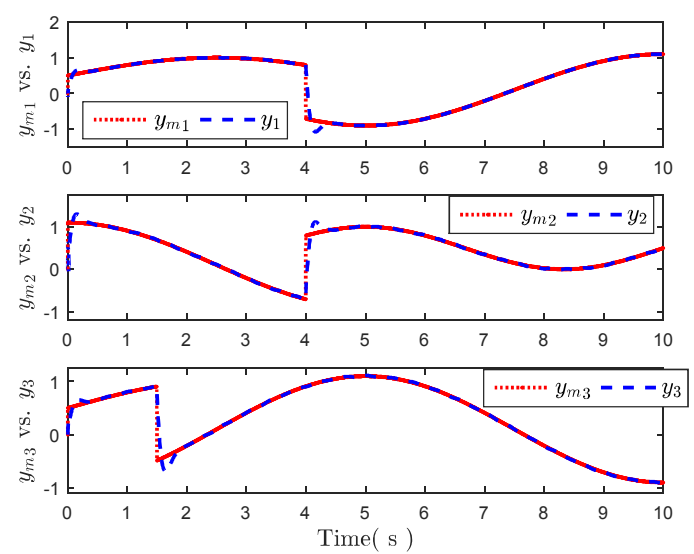

(a)
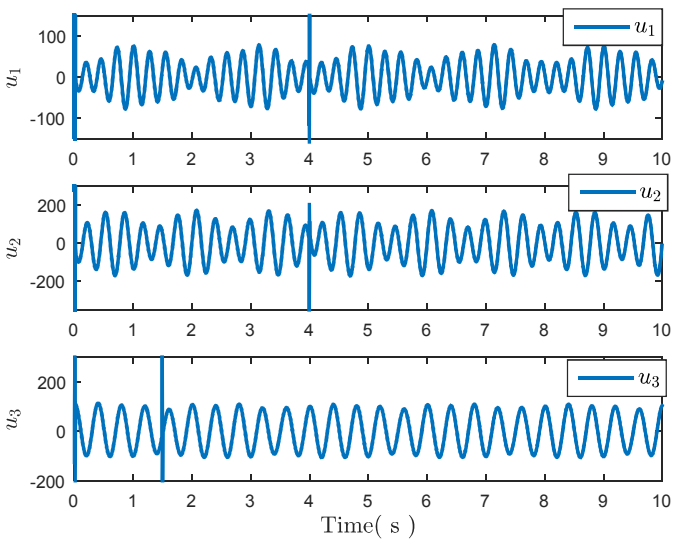

(b)

Figure 5. The closed-loop responses of the system with unknown mismatched disturbances for $i=1 \sim 3$ : (a) tracking responses $y_{i}(k)$ vs. $y_{m} i(k)$, (b) control inputs $u_{i}(k)$.

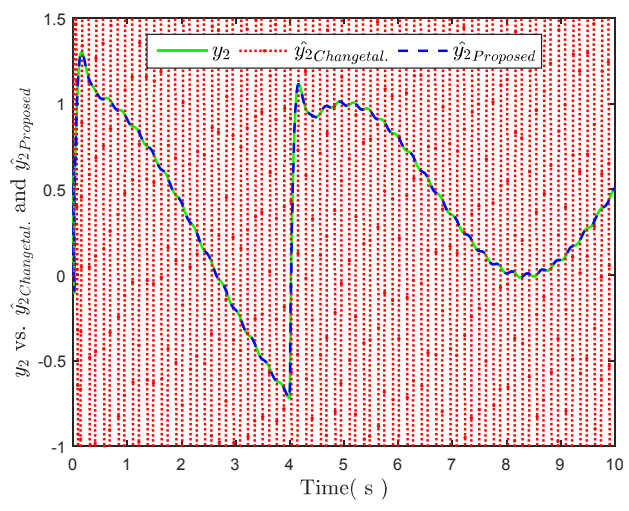

(a) 


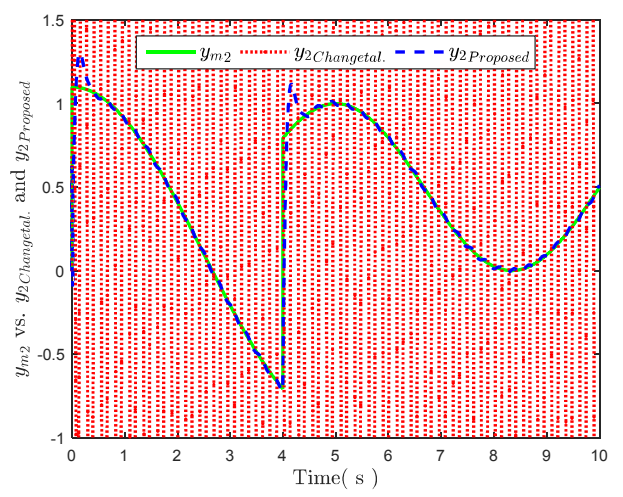

(b)

Figure 6. Comparison between [14] and the proposed method with mismatched disturbances: (a) $y_{2}(k)$ vs. $\hat{y}_{2}$ Changetal. $(k)$ and $\hat{y}_{2 \text { Proposed }}(k)$ (b) tracking responses $y_{m} 2(k)$ vs. $y_{2}$ Changetal. $(k)$ and $y_{2}$ Proposed $(k)$ (shown by parts).

Example 3: To show the unexpected situation appeared in Step 1 of Example 1 (as well as Example 2), in which one of the closed-loop eigenvalues of the observer error-dynamics system $\sigma(\bar{G}-L \bar{C})$, i.e. 0.9940 , is not optimally assigned inside a circle with a pre-specified radius $\alpha=0.07$ due to the characteristic of the challenging system given in [16], let us consider the non-minimum-phase MIMO system with unknown external disturbances in (3), where

$$
\begin{aligned}
G & =\left[\begin{array}{cccc}
0.0562 & 0 & 0 & 0 \\
0 & 0.5882 & -0.2809 & 0 \\
0 & 0.1463 & 0.1201 & 0 \\
0 & 0 & 0 & 0.7640
\end{array}\right], H=\left[\begin{array}{lll}
1 & 0 & 0 \\
0 & 2 & 0 \\
0 & 0 & 0 \\
0 & 0 & 1
\end{array}\right], \\
C & =\left[\begin{array}{cccc}
-0.5583 & 0 & 0 & 0 \\
0 & -0.1557 & 1.4949 & 0 \\
0 & 0 & 0 & -0.570
\end{array}\right],
\end{aligned}
$$

in which the open-loop system poles are $\{0.1,0.1,0.3,0.65\}$ and the 'control zeros' are $\left\{z_{1}, z_{2}, z_{3}, z_{4}\right\}=$

$\left\{1.5245,1.1756 \times 10^{-5}, 3.7786 \times 10^{-6}, 0.9021 \times 10^{-6}\right\}$. This example relaxes the challenging condition that appeared in Example 1, namely, one of the open-loop zero 0.9941 is located near 1 . Notice that feedback control does not affect the distribution of open-loop zeros.

The initial conditions and sampling time are $x(0)=\left[\begin{array}{llll}0.1 & 0 & 0 & 0.1\end{array}\right]^{T}, \hat{x}(0)=\left[\begin{array}{llll}0 & 0 & 0 & 0\end{array}\right]^{T}$, and

$T_{s}=0.0025 \mathrm{~s}$, respectively. Assume the system state is not measurable. The high-frequency unknown input disturbances are generated by

$$
d(k)=\left[\begin{array}{c}
-1.2 \cos (30 \pi k)-\sin (10 \pi k)-0.8 \cos (5 \pi k)-0.6 \sin (10 / 3 \pi k)-0.4 \cos (2.5 \pi k) \\
-1.2 \cos (15 \pi k)-\sin (5 \pi k)-0.8 \cos (2.5 \pi k)-0.6 \sin (5 / 3 \pi k)-0.4 \cos (1.25 \pi k) \\
-0.5 \cos (10 \pi k)-\sin (3 \pi k)-0.7 \cos (15 \pi k)-0.3 \sin (10 / 3 \pi k)-0.2 \cos (5 \pi k)
\end{array}\right] .
$$

For comparison, we apply the traditional optimal LQT integrated with the linear quadratic analog observer to the system to have $u_{d}(k)=-K_{d} \hat{x}_{d}(k)+E_{d} r(k+1)$, for $Q_{c}=10^{5} I_{3}$ and $R_{c}=I_{3}$. The resulting closed-loop eigenvalues are
$\sigma\left(G-H K_{d}\right)=\left\{0.6560,0.1804 \times 10^{-5}, 0.7557 \times 10^{-5}, 0.2351 \times 10^{-4}\right\} \rightarrow$ $\left\{1 / z_{1}, z_{2}, z_{3}, z_{4}\right\}$. The observer gain $L_{o}$ is obtained based on the optimal linear quadratic regulator for $Q_{0}=10^{6} I_{4}$ and $R_{o}=I_{3}$. Simulation result is given in Figure 7, which demonstrates a poor tracking performance due to the nature of a NMP system, and it cannot be improved just by the traditional state-feedback/output feedback control.

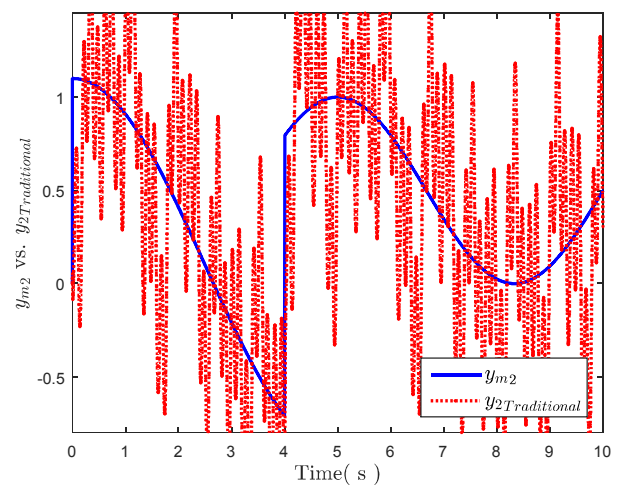

Figure 7. Tracking response of the traditional optimal state-estimate LQT controlled NMP system: $y_{m 2}(k)$ vs. $y_{2 \text { Tranditional }}(k)$ (shown by parts).

To overcome this difficulty, the design procedure is demonstrated as follows.

Step 1: Perform the optimal linear quadratic observer design.

Choose an appropriate weighting matrix pair $\left\{Q_{o}, R_{o}\right\}=\left\{10^{8} I_{3}, I_{3}\right\}$ and the radius $\alpha=0.07$ to have the observer gain $L$, which results in closed-loop eigenvalues of the observer error-dynamics system $\sigma(\bar{G}-L \bar{C})=\{0.0021 \pm 0.0012 i, 0.0054 \pm 0.0015 i$ $\left.0.0048 \pm 0.0004 i, 0.0043,-0.3144 \times 10^{-7}, 0.2794 \times 10^{-8}, 0.2084 \times 10^{-9}\right\}$. All above-mentioned open-loop eigenvalues are optimally assigned inside a circle with a pre-specified radius $\alpha=0.07$. The performance of the proposed discrete-time current output-based estimator is depicted in Figure 8. It shows that the system state $x(k)$ and unknown external disturbance $d(k)$ are well estimated, which implies $\hat{y}(t) \rightarrow y(t)$ and $\hat{d}(t) \rightarrow d(t)$ also.
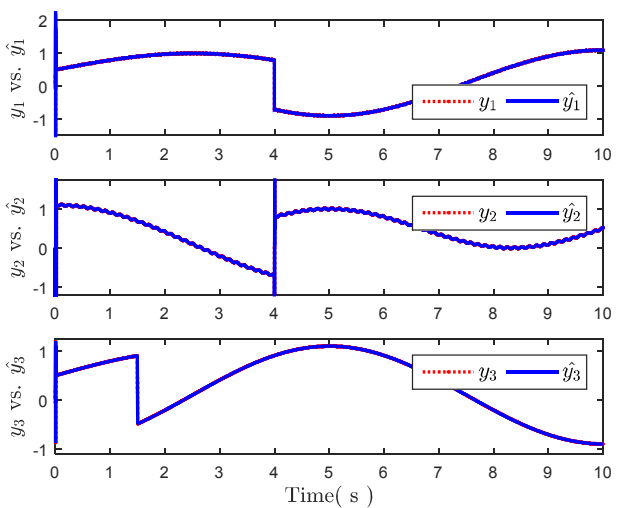

(a) 


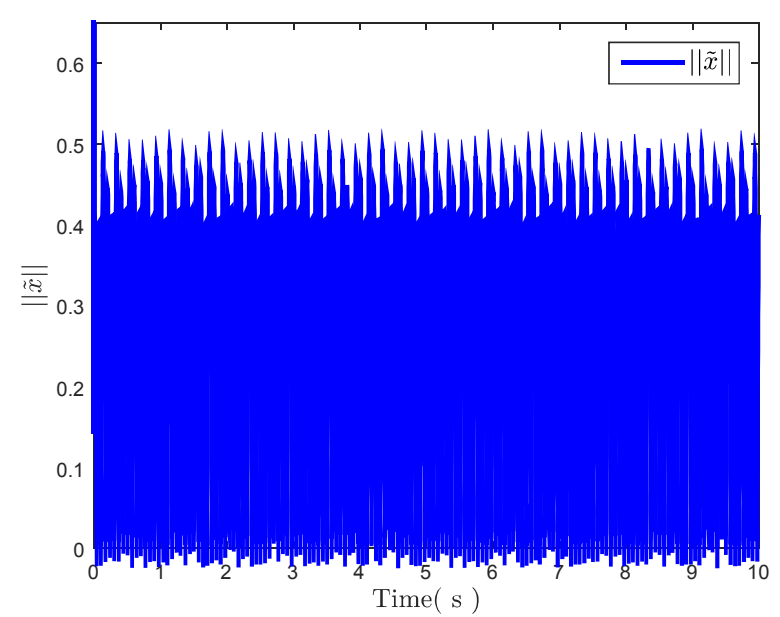

(b)
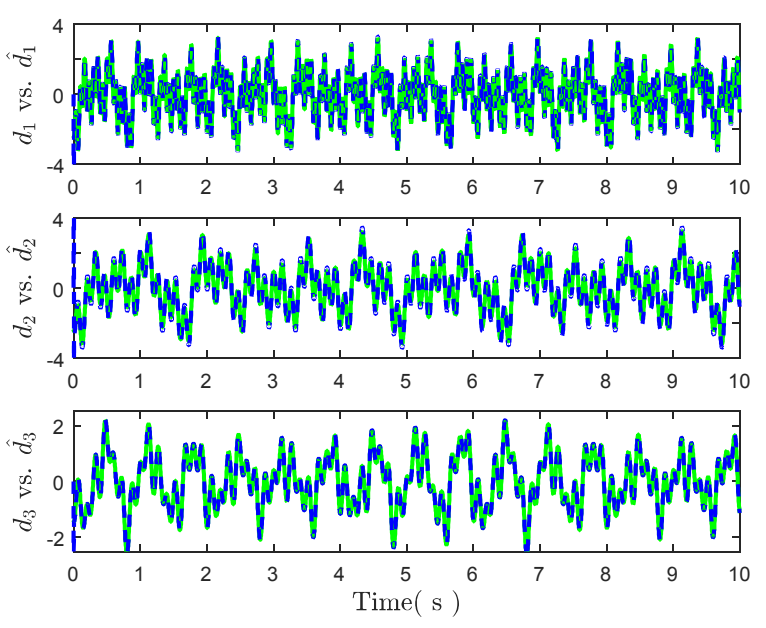

(c)
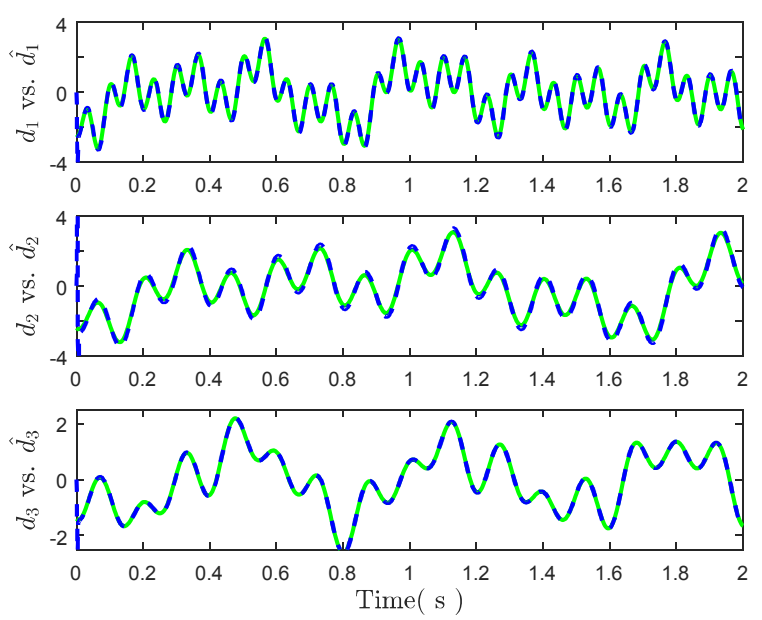

(d)

Figure 8. The closed-loop responses of the PI observer for $i=1 \sim 3$ : (a) $y_{i}(k)$ vs. $\hat{y}_{j}(k)$, (b) estimation errors of the system state; $\|\tilde{x}\|$, (c) $d_{i}(k)$ vs. $d_{i}(k)$ in global scope; $(d) d_{i}(k)$ vs. $\hat{d}_{i}(k)$ in local scope: up to 2 $s$.

Step 2: Construct the command reference model and solutions of undetermined parameter matrices.

The reference model is given in Example 2, where

$$
\begin{aligned}
u_{m}(k)= & -\left[\begin{array}{rrrr}
0.0050 & -0.0000 & -0.0000 & 0.0000 \\
0.0000 & 0.0690 & -0.0320 & -0.0000 \\
0.0000 & 0.0000 & 0.0000 & 0.0250
\end{array}\right] x_{m}(k) \\
& +\left[\begin{array}{rrr}
0.3611 & 0.0000 & -0.0000 \\
-0.0000 & -0.7408 & 0.0000 \\
-0.0000 & -0.0000 & 0.3295
\end{array}\right] r_{m}(k+1)
\end{aligned}
$$

for weighting matrices $Q_{m}=10^{5} I_{3}$ and $R_{m}=I_{3}$. Then, by given the known matrices $G_{m}, C_{m}, \Omega_{11}$, and $\Omega_{12}$ in (17), we calculate the matrices $\boldsymbol{F}, \boldsymbol{V}, \boldsymbol{W}$, and $\boldsymbol{Z}$.

Step 3: Realize the PICO-based optimal LQDT design. Realize the optimal control law

$$
u(k)=-K_{1} \hat{e}_{x}(k)-K_{2} \eta(k)-\hat{d}(k)+\boldsymbol{W} x_{m}(k)+\boldsymbol{Z} u_{m}(k) \quad \text { in }
$$

(44) for the appropriate weighting matrix pair

$\left\{Q_{z}, R_{z}\right\}=\left\{10^{6} I_{7}, I_{3}\right\}$ and the selected radius $\beta=0.15$. The eigenvalues of the closed-loop error-dynamics

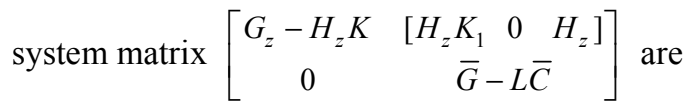

$\left\{0.1719 \times 10^{-7}, 0.1265 \times 10^{-8}, 0.5233 \times 10^{-8}, 0.0171\right.$,

$0.0212 \pm 0.0094 i, 0.0169,0.0021 \pm 0.0012 i, 0.0054 \pm 0.0015 i$ $\left.0.0048 \pm 0.0004 i, 0.0043,0.2794 \times 10^{-8},-0.3144 \times 10^{-7}, 0.2084 \times 10^{-9}\right\}$. Since $\quad \sigma\left(G_{z}-H_{z} K\right)=\left\{0.1719 \times 10^{-7}, 0.1265 \times 10^{-8}, 0.5233 \times 10^{-8}\right.$, $0.0171,0.0212 \pm 0.0094 i, 0.0169\}$ and $\sigma(\bar{G}-L \bar{C})=$

$\{0.0021 \pm 0.0012 i, 0.0054 \pm 0.0015 i, 0.0048 \pm 0.0004 i, 0.0043$, $\left.0.2794 \times 10^{-8},-0.3144 \times 10^{-7}, 0.2084 \times 10^{-9}\right\}$, it follows that the separation principle is valid and the stability is guaranteed.

The tracking response and control input of the closed-loop system are shown in Figure 9, which demonstrate that although the system is in the presence of disturbance and has an unstable zero, the system output $y(k)$ well tracks the reference trajectory $y_{m}(k)$.
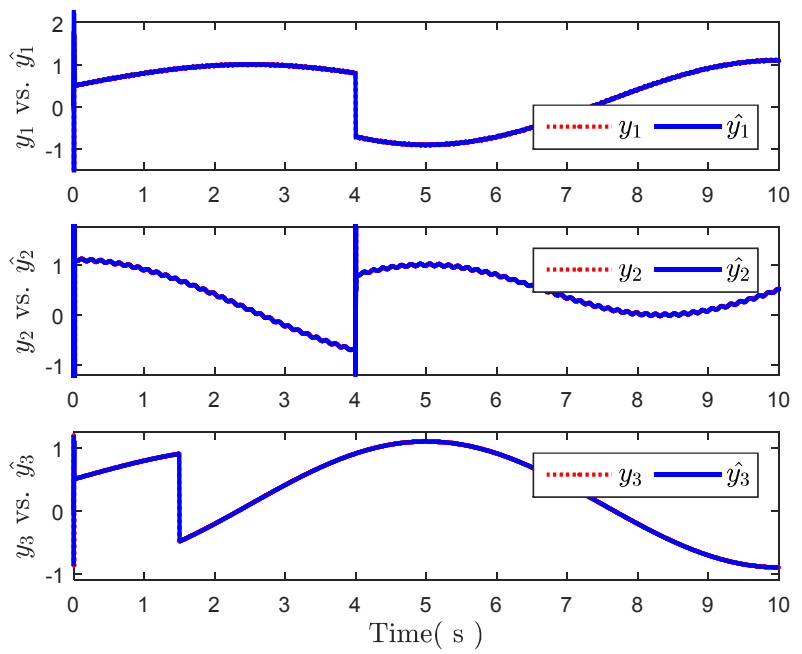

(a) 

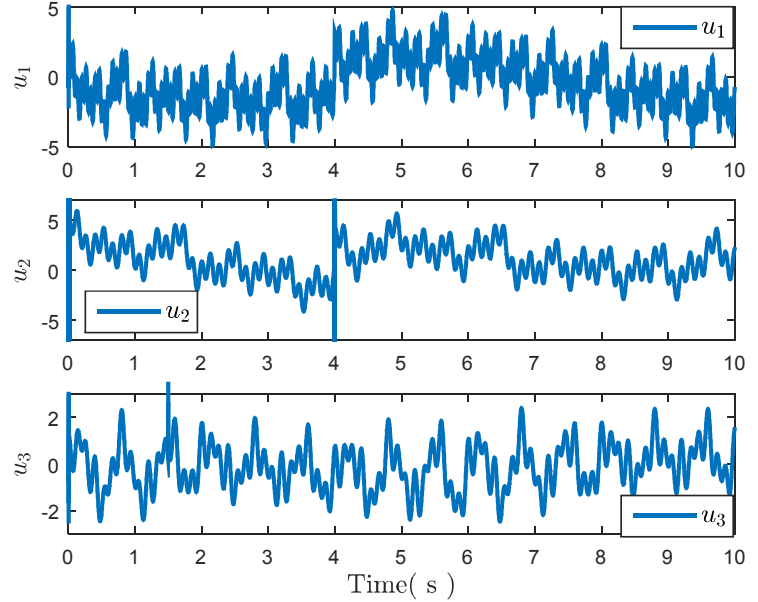

(b)

Figure 9. The closed-loop responses of the system with unknown matched disturbances for $i=1 \sim 3$ : a. tracking response $y_{i}(k)$ vs. $y_{m} i(k)$, (b) control input $u_{i}(k)$.

We compare the estimation performances between the proposed approach and that in Chang et al. method [14], where the closed-loop eigenvalues of the observer error-dynamics system in [14] are

$$
\sigma\left(\bar{G}-L_{C h} \bar{C}\right)=\{0.3780 \pm 0.1877 i, 0.6126 \pm 0.1876 i,
$$

$\left.0.3780 \pm 0.1877 i, 0.6107,0.2351 \times 10^{-4}, 0.18041 \times 10^{-5}, 0.8688 \times 10^{-6}\right\}$ for the appropriate weighting matrix pair $\left\{Q_{o}, R_{o}\right\}=\left\{10^{5} I_{10}, I_{3}\right\}$. Comparison on the estimation performances and tracking responses of these two observers depicted in Figure 10 reveals that our proposed method obviously outperforms that in [14]. This is because of the fact that the proposed approach ensures the relative stability in both the unknown input estimation and the tracking performance. The newly developed current-output observer integrated with the UID estimator-based servo design methodology can be applied to improve the performance of the discrete-time system with an unknown disturbance.

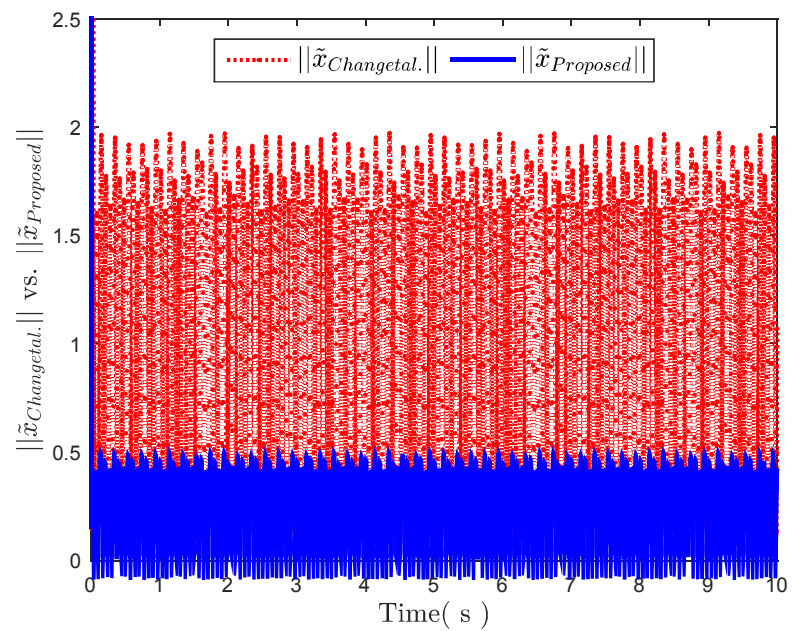

(a)

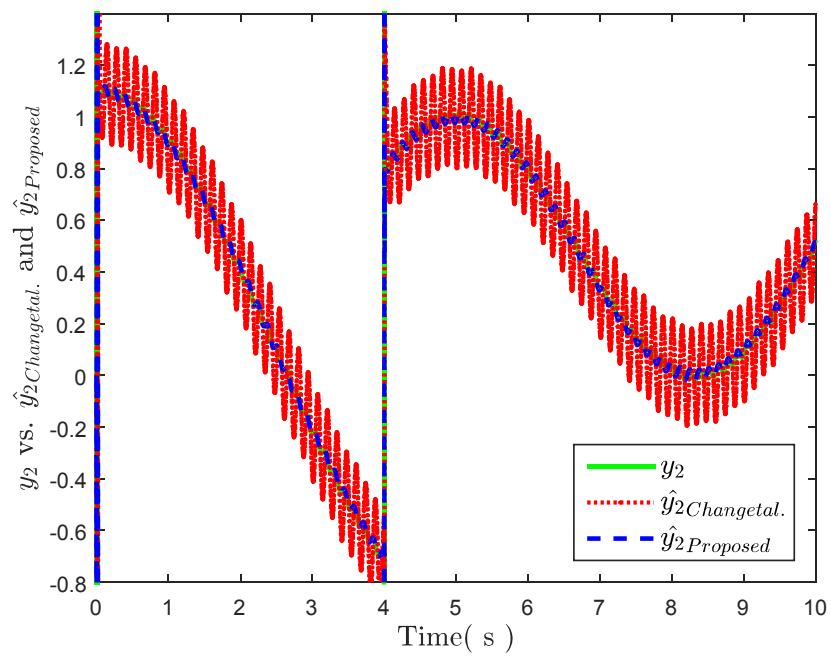

(b)

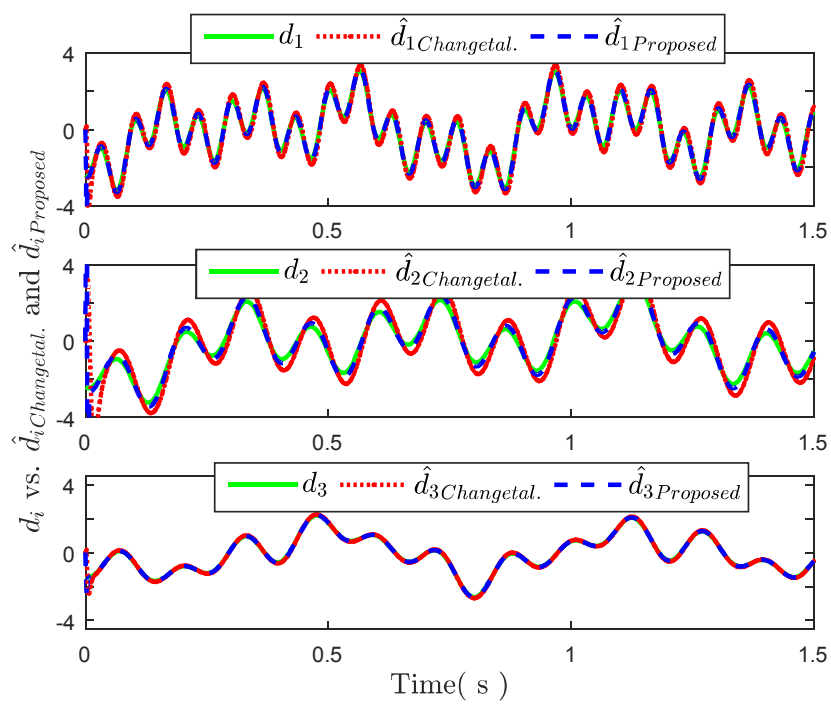

(c)

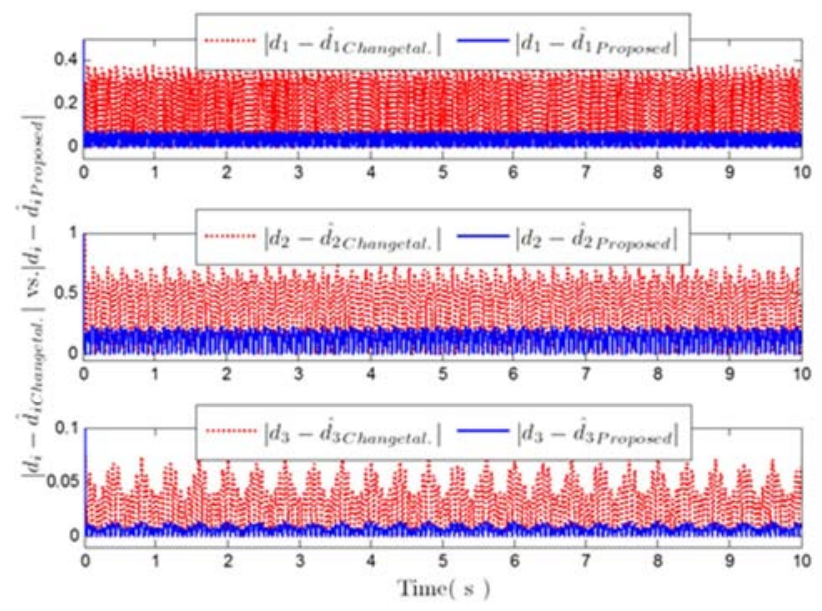

(d) 


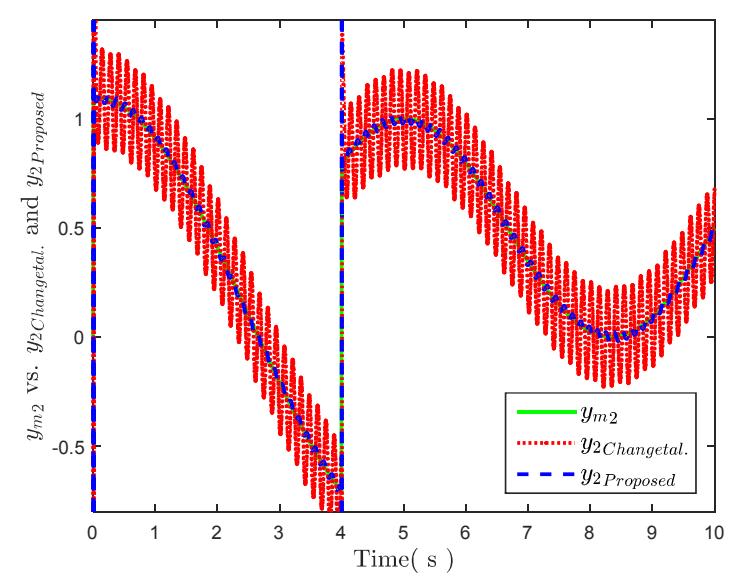

(e)

Figure 10. Comparison between [14] and the proposed method with matched

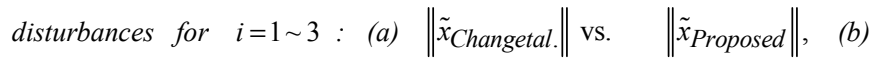
$y_{2}(k)$ vs. $\hat{y}_{2}$ Chngetal. $(k)$ and $\hat{y}_{2}$ Proposed $(k)$ (shown by parts: up to $1.5 \mathrm{~s}$ ), (c) $d_{i}(k)$ vs. $\hat{d}_{i \text { Chngetal. }}(k)$ and $\hat{d}_{i \text { Proposed }}(k)$ (shown by parts), (d)

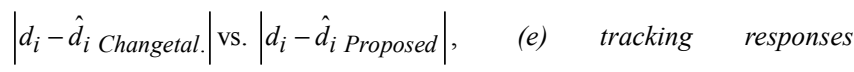
$y_{m 2}(k)$ vs. $\quad y_{2}$ Chngetal. $(k)$ and $y_{2}$ Proposed $(k)$ (shown by parts).

\section{Conclusion}

In this paper, an efficient PICO-based LQDT for non-minimum phase discrete-time system with equal input and output number, no direct-feedthrough -input matrix and unknown external disturbances has been developed. Comparing with the traditional robust optimal control, the proposed methods significantly improve the unknown input estimation and servo performance for the systems subject to the unknown disturbances. The above-mentioned objectives are achievable due to the fact that the proposed approaches ensure the relative stability in both the unknown input estimation and the servo performance from the theoretical point of view. The newly developed current-output observer integrated with the UID estimator-based servo design methodology is able to improve the discrete-time system with unknown disturbances. In addition, based on the equivalent input disturbance (EID) principle, the proposed approach is applicable to the class of systems with mismatched input disturbances. Then, numerical examples are given to show that PICO-based LQDT could rapidly track a given arbitrary reference trajectory with drastic variation. Besides, further research will be conducted to extend the proposed method in this paper to include the system with high-frequency matched and/or mismatched disturbances as well as the output disturbances.

\section{Acknowledgments}

This work was supported by the Ministry of Science and Technology of Republic of China under contracts MOST 105-2221-E-006-102-MY3 and MOST 104-2221-E-006-159-MY2.

\section{References}

[1] M. S. Chen and C. C. Chen, "Unknown input observer for linear non-minimum phase systems," Journal of the Franklin Institute, vol. 347 (2), pp. 577-588, 2010.

[2] A. Radke and Z. Gao, "A survey of state and disturbance observers for practitioners," Proceedings of the 2006 American Control Conference pp. 14-16, Minneapolis, Minnesota, 2006.

[3] H. C. Ting, J. I. Chang, and Y. P. Chen, "Proportional-derivative unknown input observer design using descriptor system approach for non-minimum phase systems," International Journal of Control, Automation, and Systems, vol. 9 (5), pp. 850-856, 2011.

[4] A. Termehchy and A. Afshar, "A novel design of unknown input observer for fault diagnosis in non-minimum phase systems," Smart Instrumentation, Measurement and Applications (ICSIMA), 2013 IEEE International Conference, pp. 1-6, Kuala Lumpur, 2013.

[5] J. H. She, M. Fang, Y. Ohyama, H. Hashimoto, and M. Wu, "Improving disturbance-rejection performance based on an equivalent-input-disturbance approach," IEEE Transactions on Industrial Electronics, vol. 55 (1), pp. 380-389, 2008.

[6] D. Tang, L. Chen, and E. Hu, "A novel unknown-input estimator for disturbance estimation and compensation," Proceedings Australasian Conference on Robotics and Automations pp.116-1-116-8. The University of Melbourne of Melbourne, Melbourne, Vic, 2014.

[7] F. Ebrahimzadeh, J. S. H. Tsai, Y. T. Liao, M. C. Chung, S. M. Guo, L. S. Shieh, and L. Wang, "A generalized optimal linear quadratic tracker with universal applications-Part 1: Continuous-time systems," International Journal of Systems Science, vol. 48 (2), pp. 376-396, 2017.

[8] F. Ebrahimzadeh, J. S. H. Tsai, M. C. Chung, Y. T. Liao, S. M. Guo, L. S. Shieh, and L. Wang "A generalized optimal linear quadratic tracker with universal applications-Part 2: Discrete-time systems," International Journal of Systems Science, vol. 48 (2), 397-416, 2017.

[9] H. Su and G. Y. Tang, "Observer-based approximate optimal tracking control for time-delay systems with external disturbances," International Journal of Systems Science, vol. 47 (12), pp. 2837-2846, 2016.

[10] J. Yang, Y. Chen, F. Zhu, and F. Wang, "Simultaneous state and output disturbance estimations for a class of switched linear systems with unknown inputs," International Journal of Systems Science, 2016, published online DOI:10.1080/00207721.2016.1144227.

[11] T. Ishiharaa and H. J. Guo, "Design of optimal disturbance cancellation controllers via modified loop transfer recovery," Systems Science \& Control Engineering: An Open Access Journal, vol. 3, pp. 332-339, 2015.

[12] W. Zhang, Y. Wang, Y. Liu, and W. Zhang, "Multivariable disturbance observer-based $\mathrm{H}_{2}$ analytical decoupling control design for multivariable systems," International Journal of Systems Science, vol. 47(1), pp. 179-193, 2015.

[13] J. L. Chang, "Applying discrete-time proportional integral observers for state and disturbance estimations," IEEE Transactions on Automatic Control, vol. 51 (5), pp. 814-818, 2006. 
[14] J. L. Chang, H. C. Ting, and Y. P. Chen, "Robust discrete-time output tracking controller design for non-minimum phase systems," Journal of System Design and Dynamics, vol. 2 (4), pp. 950-961, 2008.

[15] K Abidi, J. X. Xu, and Y. Xinghuo, "On the discrete-time integral sliding mode control," IEEE Transactions on Automatic Control, vol. 52 (4), pp. 709-715, 2007.

[16] L. P. Wang, "Model Predictive Control System Design and Implementation using MATLAB," London: Springer, 2009.
[17] F. Ding and T. Chen, "Gradient based iterative algorithms for solving a class of matrix equation," IEEE Transactions on Automatic Control, vol. 50 (8), pp. 216-1221, 2005.

[18] L. Benvenuti, M. D. Di Benedetto, and J. W. Grizzle, "Approximate output tracking for nonlinear non-minimum phase systems with an application to flight control," International Journal of Robust and Nonlinear Control, vol. 4 (3), pp. 397-414, 1994. 